\title{
Absence of full-length dystrophin impairs normal maturation and contraction of cardiomyocytes derived from human-induced pluripotent
} stem cells

\author{
J. Manuel Pioner $\oplus^{1 \dagger}{ }^{\dagger}$, Xuan Guan ${ }^{2 \dagger}$, Jordan M. Klaiman ${ }^{2}$, Alice W. Racca ${ }^{3}$, Lil Pabon ${ }^{4,5,6}$, \\ Veronica Muskheli ${ }^{5}$, Jesse Macadangdang $\oplus^{2}$, Cecilia Ferrantini ${ }^{1}$, \\ Michael R. Hoopmann $\oplus^{7}{ }^{7}$, Robert L. Moritz $\oplus^{7}$, Deok-Ho Kim ${ }^{2,6}$, Chiara Tesi ${ }^{1}$, \\ Corrado Poggesi ${ }^{1}$, Charles E. Murry $\oplus^{2,4,5,6}$, Martin K. Childers ${ }^{6,8}$, David L. Mack ${ }^{2,6,8 *}$, \\ and Michael Regnier ${ }^{2,5,6 *}$
}

${ }^{1}$ Experimental and Clinical Medicine, Div. of Physiology, University of Florence, Florence, Italy; ${ }^{2}$ Bioengineering, University of Washington, Seattle, WA, USA; ${ }^{3}$ School of Biosciences,

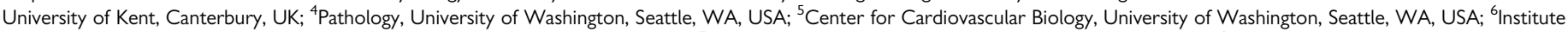

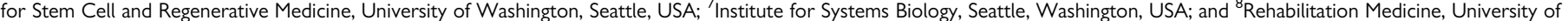
Washington, Seattle, WA, USA

Received 11 February 2019; revised 20 March 2019; editorial decision 14 April 2019; accepted 17 April 2019

Aims

Heart failure invariably affects patients with various forms of muscular dystrophy (MD), but the onset and molecular sequelae of altered structure and function resulting from full-length dystrophin (Dp427) deficiency in MD heart tissue are poorly understood. To better understand the role of dystrophin in cardiomyocyte development and the earliest phase of Duchenne muscular dystrophy (DMD) cardiomyopathy, we studied human cardiomyocytes differentiated from induced pluripotent stem cells (hiPSC-CMs) obtained from the urine of a DMD patient.

Methods

and results

The contractile properties of patient-specific hiPSC-CMs, with no detectable dystrophin (DMD-CMs with a deletion of exon 50), were compared to CMs containing a CRISPR-Cas9 mediated deletion of a single G base at position 263 of the dystrophin gene (c.263delG-CMs) isogenic to the parental line of hiPSC-CMs from a healthy individual. We hypothesized that the absence of a dystrophin-actin linkage would adversely affect myofibril and cardiomyocyte structure and function. Cardiomyocyte maturation was driven by culturing long-term (80-100 days) on a nanopatterned surface, which resulted in hiPSC-CMs with adult-like dimensions and aligned myofibrils.

Conclusions

Our data demonstrate that lack of Dp427 results in reduced myofibril contractile tension, slower relaxation kinetics, and to $\mathrm{Ca}^{2+}$ handling abnormalities, similar to DMD cells, suggesting either retarded or altered maturation of cardiomyocyte structures associated with these functions. This study offers new insights into the functional consequences of Dp427 deficiency at an early stage of cardiomyocyte development in both patient-derived and CRISPRgenerated models of dystrophin deficiency.

Keywords

Human iPSC-cardiomyocytes • Muscular dystrophy • Dystrophin • Myofibrils • CRISPR-Cas9 genome editing

\section{Introduction}

Dilated cardiomyopathy and congestive heart failure are leading causes of death in patients with Duchenne muscular dystrophy (DMD). In young patients, DMD cardiomyopathy is characterized by initial diastolic dysfunction and ventricular dilation leading to the onset of clinical manifestations. ${ }^{1}$ Therefore, there is considerable interest in determining the pre-symptomatic aetiology and the downstream development of cardiac abnormalities resulting from an absence of dystrophin in the heart.

\footnotetext{
* Corresponding authors. E-mail: dmack21@uw.edu (D.L.M.); E-mail: mregnier@uw.edu (M.R.)

† The first two authors contributed equally to the study.

Published on behalf of the European Society of Cardiology. All rights reserved. @ The Author(s) 2019. For permissions, please email: journals.permissions@oup.com.
} 
It is widely accepted that dystrophin deficiency results in increased cellular vulnerability to mechanical stress during muscle contraction. However, it is not known how this may affect the contractile apparatus and, in turn, excitation-contraction (EC) coupling mechanisms of the myocardium. Full-length dystrophin (Dp427) is strategically placed to carry out mechanical roles, such as maintenance of membrane stability and transduction of mechanical force from and to the extracellular matrix, acting as a shock absorber to protect the sarcolemma. Dystrophin also interacts with the dystrophin glycogen complex (DGC), which has been shown to regulate signalling pathways involved in $\mathrm{Ca}^{2+}$ homeostasis, mitochondrial function, motor protein interaction, and musclespecific gene expression. ${ }^{2}$ It has been proposed that a distinct pool of dystrophin is co-localized with $\alpha$-actinin and desmin at the Z-disks of myofibrils in cardiac, but not in skeletal muscle. ${ }^{3}$ In addition, a short splice variant of dystrophin (Dp71) expressed in cardiac muscle is specifically located in transverse ( $\mathrm{T}$ )-tubular membranes, though its role is not clear. $^{4}$

The absence of the damping role of Dp427 may negatively impact the contractile apparatus and function of sarcomeres, the contractile units that compose myofibrils, from a very early stage of development. Observations of cardiac samples from DMD paediatric patients suggest that lack of dystrophin may interfere with myofibril formation during development. ${ }^{5}$ However, little is known about the role of dystrophin during human cardiac development. Previous studies found dystrophin expression on the surface of cardiomyocytes from 8-week-old foetuses. ${ }^{6,7}$ Curiously, pre-myofibrils begin to assemble at the cell edges and fuse at the level of the Z-bodies to form mature myofibrils that compose the primitive contractile machinery of cardiomyocytes. ${ }^{8}$ From 8 to 24 weeks gestation dystrophin begins to distribute homogeneously, ${ }^{6}$ together with increased myofibril bundling, during the time when there is a shift from $\alpha$ - to slow $\beta$-myosin heavy chain $(\mathrm{MHC})$ in ventricular myocardium. ${ }^{9,10}$ Although little information of this mechanism has been reported for cardiac muscle, skeletal myotube fibre thickening is associated with increased density of dystrophin, together with downregulation of foetal myosin and upregulation of adult myosin isoforms. ${ }^{6,11}$ At the Zdisk level, costameree proteins connect Z-bands to $T$ tubules and it is here that dystrophin can transmit biomechanical stress through associated signalling proteins required for myofibril maturation. ${ }^{12}$

Cardiomyocytes from patients, generated from human cardiomyocytes differentiated from induced pluripotent stem cells (hiPSC-CMs), offer a limitless source of cells or cardiac tissue for modelling foetal development of cardiomyopathies in vitro. We recently reported that hiPSC-CMs benefit considerably from being cultured long term on nanopatterned surfaces, ${ }^{13,14}$ with dramatic improvement of myofibril assembly and alignment, together with preliminary formation of caveolae/ T-tubule-like profiles. However, myofibrils from these hiPSC-CMs still have contractile properties very similar to what we recently reported for myofibrils from foetal heart tissue of 12-14 weeks of gestational age. ${ }^{10,14}$ Here, we report experiments using hiPSC-CMs generated from the urine of a DMD patient with a total absence of dystrophin, due to a deletion of exon 50 in the dystrophin gene (DMD- $\Delta$ Exon50). To minimize the impact of underlying genetic heterogeneity within patients, the current study reports the creation of a Dp427 null allele by deleting a single $\mathrm{G}$ base using the CRISPR-Cas9 enzyme (c.263delG) isogenic to a healthy control. Compared to its parental isogenic control, the c.263delG-cell line lacks expression of Dp427 but has low levels of a shorter truncated dystrophin with complete deletion of the actin binding domain (ABD). Thus, this represents a second cell line with full-length dystrophin deficiency. Our data definitively demonstrate that the Dp427 null mutation alone impairs cardiomyocyte physiology. We previously reported that at earlier stages cardiac differentiation, both DMD and c.263delG cell lines consistently displayed unique features such as increased membrane susceptibility to hypotonic stress, slower $\mathrm{Ca}^{2+}$ transients, and faster mitochondrial permeability pore opening time. ${ }^{13,15}$

In this study, we hypothesized that sarcomere dysfunction was a major consequence of mechanical and structural changes caused by lack of Dp427. We isolated myofibrils from DMD-hiPSC-CMs to study tension generating capacity and the kinetics of activation and relaxation, using our recently reported method. ${ }^{14}$ We identified contractile dysfunction at the myofibril and single cell level for DMD-hiPSC-CMs compared with control-hiPSC-CMs, and found similar results with the isogenic (c.263delG) cell line generated from the control-hiPSCs. This demonstrates directly that lack of full-length dystrophin alone is sufficient to precipitate structural alterations and impact both myofibril function and $\mathrm{Ca}^{2+}$ handling early in cardiomyocyte development. The results of this and future investigations of contractile properties will help to dissect mechanisms of the earliest stages of dystrophin deficiency that can lead to MD cardiomyopathy and suggest interventions that could correct or delay the cardiomyopathy at the pre-symptomatic stage.

\section{Methods}

An expanded version of the materials and methods appears in the Supplementary material online that accompanies this manuscript. All authors provided informed, written consent prior inclusion in the study.

\section{Human pluripotent stem cell from urine and cardiac differentiation}

Protocols for this study were approved by the Institutional Review Board of the University of Washington. Informed consent was given by patients prior to the inclusion of people in the study. The isolation of the human cells and the subsequent reprogramming into iPSC lines was performed conform the declaration of Helsinki. Urine-derived cells from a healthy male donor (control UC3-4 A1) and a DMD patient with deletion of exon 50 in DMD gene (UC72039) were reprogrammed into hiPSC lines as previously described. ${ }^{15}$ An isogenic control (c.263delG, UC1015-6) cell line was generated from control cell line by CRISPR-Cas9-mediated deletion of a single frame at the $5^{\prime}$ of Exon1 in DMD gene. Differentiated cardiomyocytes were obtained as detailed in the Supplementary material online and matured long-term as single cells on nanotopographic (nanopattern) substrata. ${ }^{14}$

\section{Construction of Cas9/guide RNA-expressing vectors}

Guide sequences targeting the $3^{\prime}$-end of the first muscle-specific exon of human dystrophin were designed using the CRISPR design tool (http://crispr. mit.edu/) supported by the Zhang lab at $\mathrm{MIT}^{16}$ (see Supplementary material online, Materials and methods section for full description). Functional validation of the guide sequences was carried out by transfecting HEK293 cells with hSpCas9 (BB)-2A-Puro plasmids. The DNA dystrophin locus was PCR amplified by Phusion ${ }^{\circledR}$ High-Fidelity hot start II DNA Polymerase (Thermo, Waltham, MA, USA) using primers shown in Supplementary material online.

\section{Genome targeting and cell cloning}

hSpCas9 (BB)-2A-Puro incorporated guide sequence 4 was chosen to transfect normal male iPSCs (UC3-4) to generate the DMD-c.263delG cell line. After Versene dissociation (Life Technologies), $3 \times 10^{6}$ UC3-4 iPSCs (generated from a normal donor) were transfected using the Neon Transfection System (Life Technologies, CA, USA) following manufacturer's instructions. Genomic DNA of individual colonies was extracted for PCR amplification of 
the targeted dystrophin region. Mutation of the dystrophin locus was confirmed by sequencing and ion chromatogram $(\mathrm{XIC})$ analysis (details in the Supplementary material online).

\section{Cell morphology, myofibril structural, and protein analysis}

Long-term cultured hiPSC-CM on nanopatterned surfaces were stained for $\alpha$-actinin, dystrophin, caveolin 3 (Cav3), and DAPI for morphological characteristics and assessed by confocal microscopy. Myofibril structure was assessed by transmission electron microscopy (TEM). Sarcomere protein composition was studied by SDS-PAGE and western blot analysis using frozen remains of myofibril preparations used for contractile assessments. Details are provided in the Supplementary material online.

\section{Myofibril mechanics}

Myofibrils were isolated from DMD-hiPSC-CMs by a recently described cell skinning and purification procedure. ${ }^{14}$ Myofibril mechanics, kinetics, and $\mathrm{Ca}^{2+}$ sensitivity $\left(\mathrm{pCa}_{50}\right)$ were measured by fast solution switching in a custom-built apparatus described in previous publications. ${ }^{10,14,17-19}$ Details are provided in the Supplementary material online.

\section{Cell shortening and $\mathrm{Ca}^{2+}$ transients}

Single hiPSC-CMs were visualized for fractional shortening using an inverted microscope coupled to a video-based edge detection system with synchronously detection of Fura-2 AM fluorescence for measuring intracellular $\mathrm{Ca}^{2+}$ content. Cells were perfused at $37^{\circ} \mathrm{C}$ with Tyrode solution and paced at 0.5 , 1 , or $2 \mathrm{~Hz}$ by field stimulation. Details are provided in the Supplementary material online.

\section{Statistical analysis}

All data are reported in means \pm SEM and were compared using a one-way, two-way analysis of variance (ANOVA) with a Tukey post hoc test with statistical significance set at $P<0.05^{*}$ and $P<0.01 * *$ were applied for differences in means between groups/conditions. For each analysis, $n$ represented number of cells, myofibrils, or sarcomere determinants. Total number of cell differentiation runs $(N)$ used for this work were for Control $N=6, D M D N=5$, c.263delG $N=3$. The number of individual experiments (individual cover slides) for each assessment was at least 3 from different differentiation runs.

\section{Results}

\section{Human iPSC-derived cardiomyocytes harbouring a single base pair deletion (c.263delG) fail to express Dp427}

Control-hiPSC (UC3-4 A1) and DMD-hiPSCs (UC72039 D3) were generated from urine-derived cells of a healthy volunteer and from a patient carrying a deletion of exon 50 ( $\Delta$ Exon 50$)$ in the dystrophin gene. ${ }^{15}$ To create an isogenic hiPSC line, the dystrophin gene of a normal male iPSC line was targeted by the CRISPR-Cas9 enzyme at the $5^{\prime}$ coding region within exon 1 of Dp427m (Figure 1A), the predominant dystrophin isoform of heart muscle. One clone revealed a deletion of guanine 263 (c.263delG) (Figure 1B), shifting the reading frame to prematurely terminate dystrophin translation. To confirm that direct genomic manipulation did not elicit undesirable chromosomal instability, the c.263delG clone was re-examined for normal stem cell morphology, expression of pluripotency-related markers (Supplementary material online, Figure S1A), and a normal male karyotype (Supplementary material online, Figure S1B).

The absence of dystrophin protein was measured by western blot (Figure 1C, Supplementary material online, Figure S1C); antibodies against the C-terminal domain of dystrophin to detect the $427 \mathrm{kDa}$ dystrophin protein in mutant cardiomyocytes, indicating that a full length dystrophin-null may not have created by CRISPR-Cas9 methodology. However, proteomic analysis by mass spectroscopy revealed a truncated form of dystrophin with deletion of the $\mathrm{N}$-terminal region up to exon 6 corresponding to the actin-binding domain of full-length dystrophin. The full sequence analysis is reported in Supplementary material online, Figure S2 and summarized in Figure 1D. The gel could not discern the size difference between full-length and the truncated form (schematic Figure 1D). The observed signal by ion chromatogram (XIC) analysis of a peptide from the $\mathrm{N}$-terminal region (LLDLLEGLTGQK), is visible only in the healthy control (Supplementary material online, Figure S3A) but absent in the c.263delG mutant (Supplementary material online, Figure S3B-F) (Details in the Supplementary material online).

\section{DMD-hiPSC-CM dimensions and myofibril organization}

Following cardiac differentiation with a monolayer directed protocol, ${ }^{20}$ 15 days post-differentiated hiPSC-CMs were re-plated and cultured on surfaces with $800 \mathrm{~nm}$ grooves/ridges (nanopatterns) for an additional 65-85 days (80-100 days total), as previously described. ${ }^{14}$ As described below, long-term culture on nanopatterns that provided topographical cues resulted in cardiomyocytes with morphological features much more mature than those from our previous reports with these cells.

We compared the dimensions of DMD-CMs with those of controlCMs in cells stained for Z-bands (anti $\alpha$-actinin) (Supplementary material online, Table S1). DMD-CMs had a significantly greater cell length (DMD: $140.23 \pm 9.58 \mu \mathrm{m}$ vs. control: $116.91 \pm 6.18 \mu \mathrm{m}, P<0.05)$ and cell diameter (DMD: $27.75 \pm 0.93 \mu \mathrm{m}$ vs. control: $17.79 \pm 0.62 \mu \mathrm{m}, P<0.01$ ), resulting in a large increase in cross-sectional area (DMD: 13, $825 \pm 2$, $548 \mu \mathrm{m}^{2}$ vs. control: $\left.7,696 \pm 519 \mu \mathrm{m}^{2}, P<0.01\right)$. The aspect ratio for both groups was similar to adult cardiomyocytes and Z-disks were observed across the entire cell width (diameter), suggesting DMD-CMs experienced hypertrophy and a greater number of myofibrils in parallel (Figure 2A). Fluorescence antibody staining of $\boldsymbol{\alpha}$-actinin was used to visualize Z-bands and the density and regularity of the transverse striation patterns were analysed with fast Fourier transform (FFT) analysis (Supplementary material online, Figure S4). The area of the first peak of the power spectrum demonstrated that DMD-CMs had the same myofibril alignment index as control-CMs (DMD: $0.018 \pm 0.006$ vs. control: $0.014 \pm 0.002, P=0.54)$. Resting sarcomere lengths were also not different from control cells (SL, DMD: $1.93 \pm 0.03$ vs. control: $1.94 \pm 0.11 \mu \mathrm{m}$, $P=0.88)$ and similar to values reported for adult cardiomyocytes, ${ }^{21}$ suggesting no active crossbridge cycling at rest (Figure 2B). Thus, at the light microscope level, myofibril and sarcomere organization did not appear to be disrupted by a lack of dystrophin when cells received topographical cues via long-term culture on nanopatterned surfaces.

\section{Loss of Dp427 impairs myofibril mechanics and kinetics in cardiomyocytes}

We characterized the contractile properties of myofibrils isolated from DMD cell lines for comparison with the control UC3-4 A1 cell line and a second hiPSC-CM cell line reported in our recent work. ${ }^{14}$ Briefly, hiPSC-CMs were chemically demembranated using rigor solution with $1 \%$ Triton to obtain single or small bundles (2-5 myofibrils per bundle). The mechanical and kinetic properties of DMD- and control-myofibrils were measured in a custom-built apparatus using fast solution switching technique, as previously described. ${ }^{10,14,17}$ Figure $3 \mathrm{~A}$ demonstrates that 


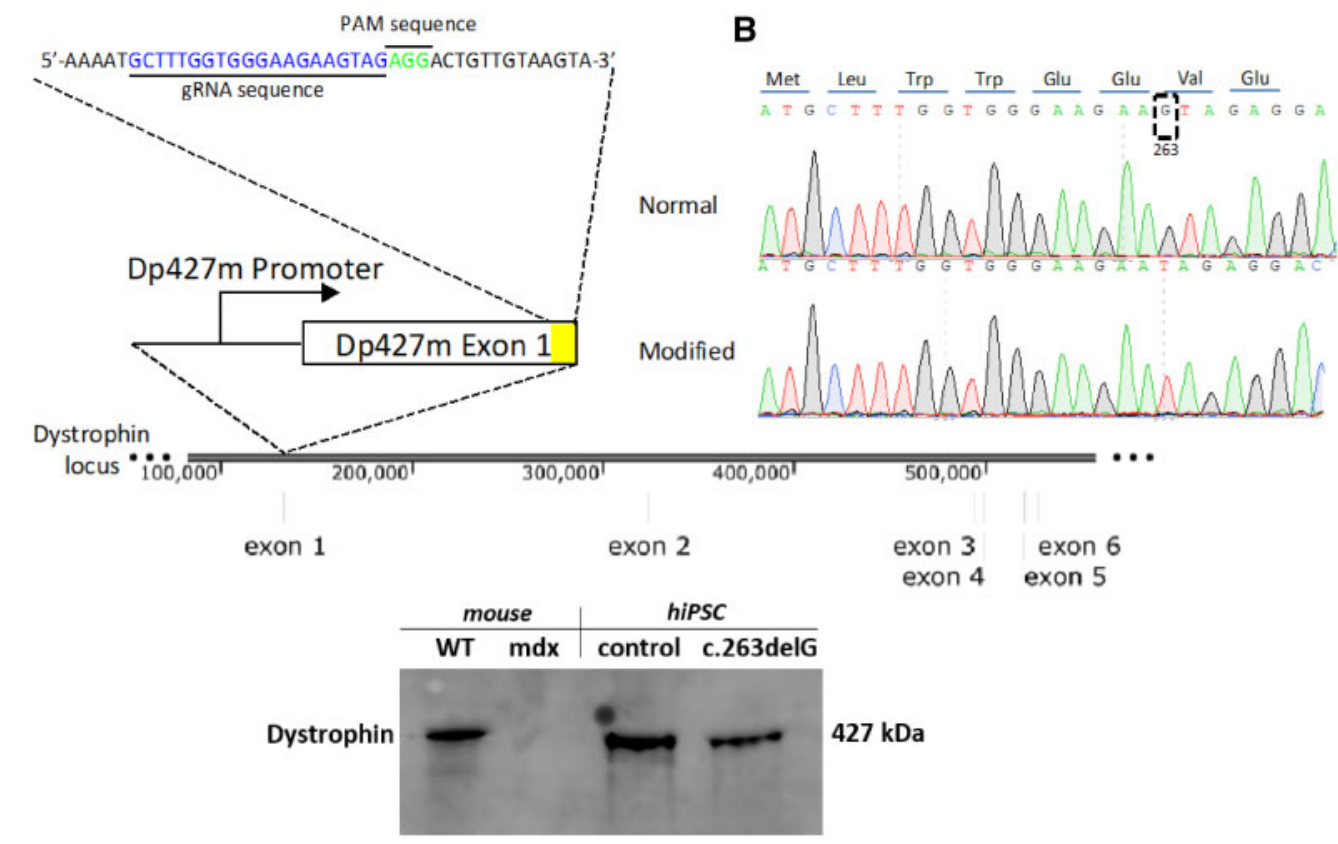

A
B

D

C

.

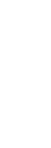

.

(1) 

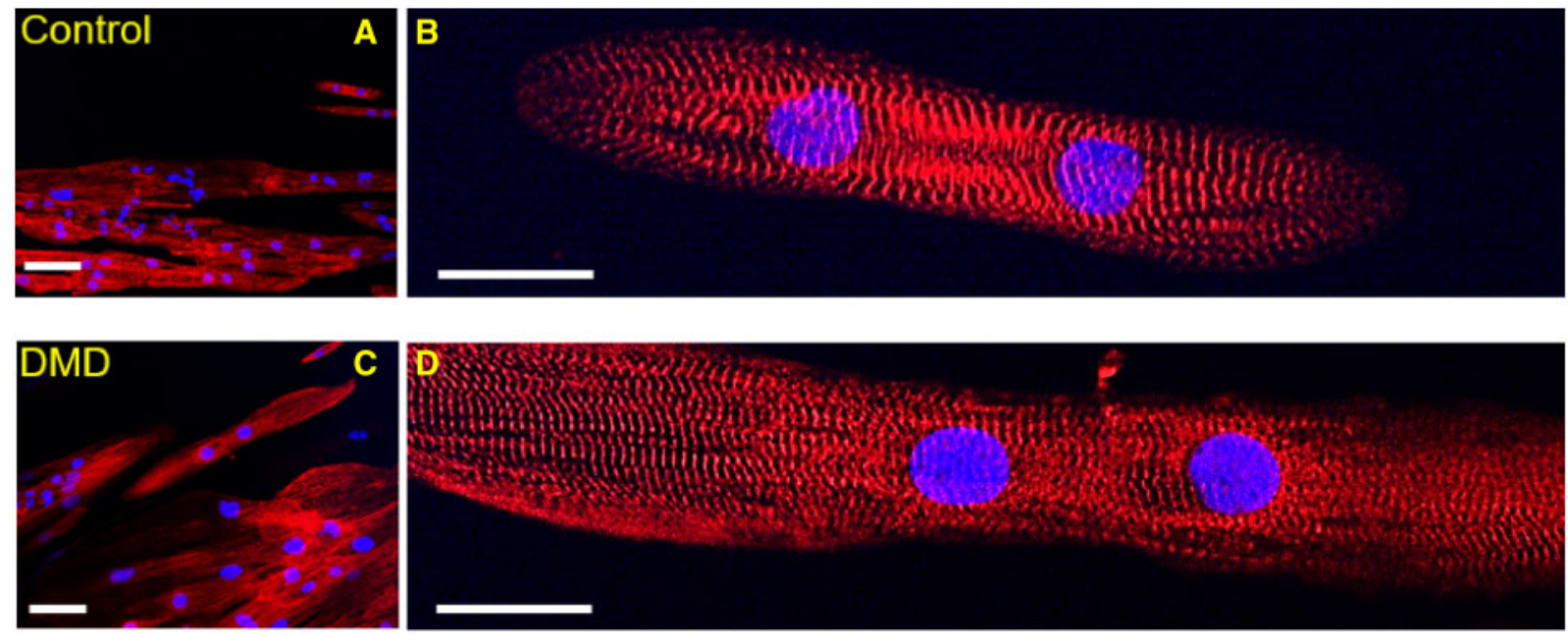

Figure 2 DMD-CM dimensions, myofibril alignment and sarcomere length. Representative images of Control- and DMD-CMs stained for Z-bands ( $\alpha$ actinin, red) and nuclei (DAPI, blue) for measuring cell dimensions and myofibril organization at $20 \times$ and $60 \times$ of magnification images of control- $(A, B)$ and DMD-CMs $(C, D)$. Quantitative analysis are reported in the results: cellular cross section and cell diameter $\left(n_{\text {control }}=159, n_{D M D}=135\right) ; m y o f i b r i l$ alignment index $\left(\mathrm{n}_{\text {control }}=20, \mathrm{n}_{\mathrm{DMD}}=30\right)$. Resting sarcomere length $(\mathrm{SL})\left(\mathrm{n}_{\text {control }}=20, \mathrm{n}_{\mathrm{DMD}}=32, P=0.88\right)$. $P$-value was estimated by one-way ANOVA with Tukey post hoc test. $N=3$ per cell line. Scale bars equal to $20 \mu \mathrm{m}$ for $(A)$ and $(C)$ and $40 \mu \mathrm{m}$ for $(A),(D)$.

High-magnification images $(40000 \times)$ confirmed clearly aligned myofibril Z-bands with widely spaced thick filaments likely forming bundles (Figure 4A). Figure 4B summarizes a quantitative evaluation of sarcomere features of DMD- and control-CMs. Resting sarcomere length measured from TEM images was shorter compared to measurements obtained from confocal images for all cell lines, likely due to cell shrinkage during the fixing process, but no difference was found between DMD and control-CMs using either method (with TEM, SL $L_{D M D}=$ $1.70 \pm 0.02$ vs. $\left.\mathrm{SL}_{\text {control }}=1.75 \pm 0.02 \mu \mathrm{m}, P=0.15\right)$. DMD-CM sarcomeres had greater variability in structural organization. The percentage of identifiable l-bands in sarcomeres per myofibril was significantly less for DMD-CMs $\left(I_{D M D}=36 \% \pm 8 \%\right.$ vs. $\left.I_{\text {control }}=72 \% \pm 7 \%, P<0.05\right)$. Also, sarcomere diameters (estimated from the length of Z-bands) were significantly smaller $\left(Z_{\mathrm{DMD}}=485.58 \pm 27.21 \mathrm{vs} . Z_{\text {control }}=\right.$ $645.98 \pm 34.02 \mathrm{~nm}, P<0.01$ ), suggesting a possible reduction in the parallel assembly of myofilaments within individual myofibrils. Furthermore, DMD A-bands were slightly shorter $\left(A_{D M D}=\right.$ $1226.79 \pm 31.82$ vs. $\left.A_{\text {control }}=1303.58 \pm 24.76 \mathrm{~nm}, P<0.05\right)$. $\mathrm{H}$-zones and $M$-lines were barely visible in any of the cell lines, not sufficient for quantitative measurement and statistical analysis. Combined, these data suggest that the ultrastructure of myofibrils is less developed in DMD-CM sarcomeres. This likely contributes to the deficit in tension production and, perhaps, slower relaxation.

We then examined sarcomere protein expression. Analysis was limited, due to the low amount of contractile material remaining from preparations used for functional analysis, but western blots revealed that at this time in culture (80-100 days post-differentiation) $\beta-\mathrm{MHC}$ was predominant and there was no detectable expression of $\alpha-\mathrm{MHC}$ (Figure $3 C$ ). This agrees with our recent report of hiPSC-CMs from a patient with a MYH7 mutation and control cell lines for a similar time in culture on nanopatterned surfaces. ${ }^{14}$ Also as in our previous report, we detected a mixture of slow skeletal troponin I (ssTnl) and adult cardiac troponin I
(cTnl) that appeared to be similar for all cell lines. A quantitative analysis of the ratio of ssTnl to $\mathrm{cTnl}$ is needed as there may be some difference between the two cell lines (Figure 4C, Supplementary material online, Figure S6) that may account for the greater $\mathrm{Ca}^{2+}$ sensitivity of force in DMD CMs (Figure 3E). An important point to make is that cTnl composition is $\geq 50 \%$ of total Tnl. This is much greater than what has been reported for most hiPSC-CM studies. The switch to $\beta$-myosin and presence of significant amounts of $\mathrm{cTnl}$ demonstrate maturation of all the $\mathrm{CM}$ lines, and the similarities in their composition in myofibrils suggest the lower tension production and slower relaxation kinetics are not likely due to differences in these proteins.

\section{Loss of Dp427 prolongs cardiac cell contractility}

To examine how dystrophin deficiency impacts intact cell contractile properties, we measured fractional shortening and relaxation during stimulated contractions using the lonOptix edge detection system at $37^{\circ} \mathrm{C}$ in the presence of $1.8 \mathrm{mM}$ extracellular $\mathrm{Ca}^{2+}$ (Figure $4 \mathrm{~A}$ ). Cells were paced at $0.5,1$, and $2 \mathrm{~Hz}$, while continuously perfused with Tyrode solution. Fractional shortening at the peak of the twitch (expressed as the percentage of variation from total diastolic cell length) is commonly used as a parameter that reflects contractile capacity at low loads. The fractional shortening of DMD-CMs was not significantly different from control-CMs at any pacing frequency (Figure 4B). This would seem to suggest no deficits in contractility at low load for dystrophin deficient cells. However, the speed of unloaded contraction was reduced in both DMD- $\Delta$ Exon50 and c.263delG-CM lines. Cell shortening (time to peak of contraction; DMD: $P<0.01$, c.263delG: $P<0.01$; Figure $4 C$ ) was slower at $2 \mathrm{~Hz}$ and relaxation (time from peak contraction to $50 \%$ of relaxation RT50; $P<0.01$ for both cell lines; Figure 4D) was slower at all stimulation frequencies. This indicates that both DMD- $\Delta$ Exon 50 and c.263delG cell lines had distinctive prolonged contraction and relaxation after 
A
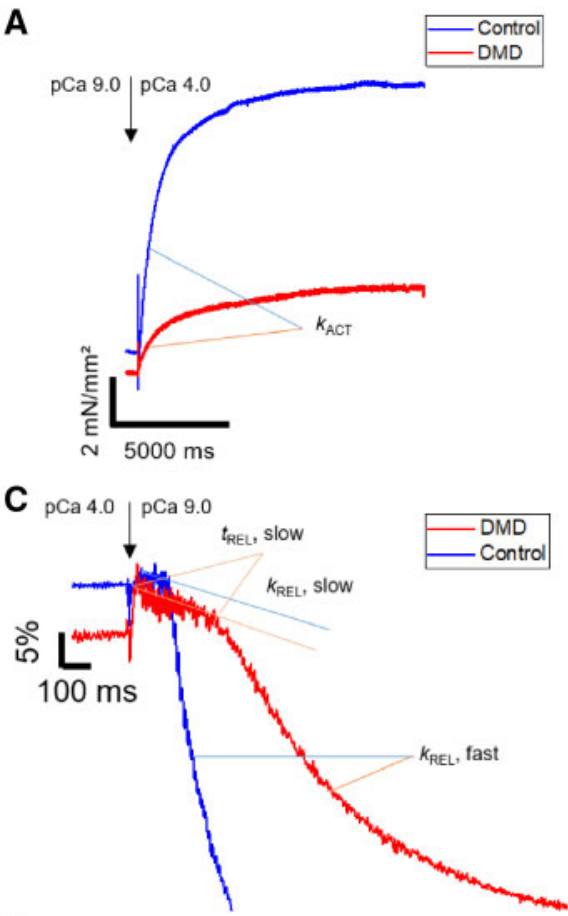

E

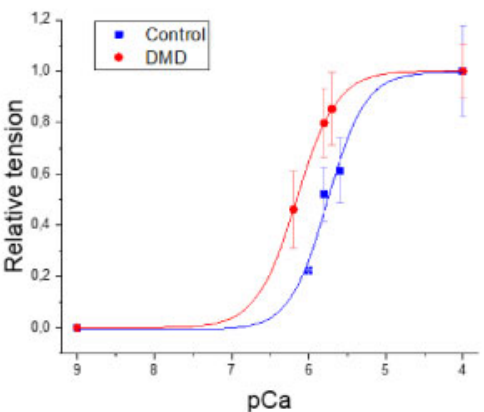

B

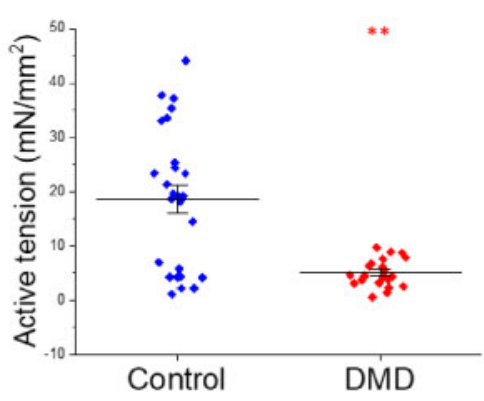

D

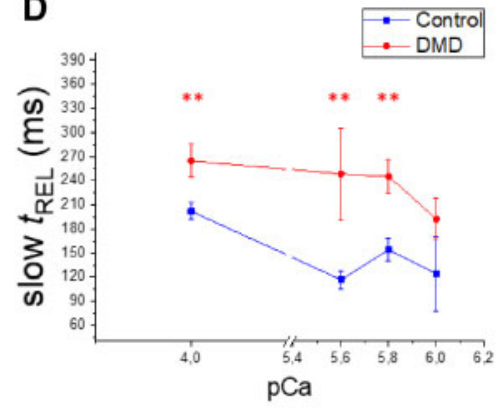

$\mathbf{F}$

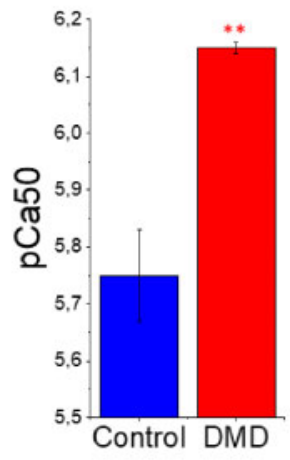

Figure 3 Mechanical properties of myofibrils from DMD-CMs. Mechanical and kinetic properties of control- (UC3-4 A1) and DMD-CM myofibrils were measured in a custom-built apparatus using fast solution switching. Compared to control-, DMD-CM myofibrils had ( $A$, B) lower maximal (pCa4.0, representative traces) tension generating capacity. Kinetics of activation ( $\left.k_{\mathrm{ACT}}\right)$ and $(C)$ relaxation (slow $k_{\mathrm{REL}}$ ) were analogous to control-CM myofibrils. $A$ slower fast phase of relaxation (fast $k_{R E L}$ ) with prolonged duration of slow phase of relaxation (slow $t_{R E L}$ ) correlated to $\mathrm{Ca}^{2+}$ concentration in the $(D) t_{R E L}$ slow/pCa plot. (E) Percentage of tension was plotted against relative $\mathrm{pCa}$ level and the Hill equation was used to determine $(F) \mathrm{myofibril} \mathrm{Ca}^{2+}$ sensitivity $\left(\mathrm{pCa} \mathrm{a}_{50}\right)$. Data for individual myofibrils are reported in Table $1 ; * * P<0.01$ estimated by one-way ANOVA with Tukey post hoc test. $N=3$ for each cell line. Number of experiments control $=10$ and DMD $=5, n$, number of single myofibrils are reported in Table 1.

long-term culture. Coupled with results from myofibril measurements, we conclude that slower relaxation is at least partially due to properties of the contractile apparatus. All cell lines had a low but residual population of spontaneously beating cells that were excluded from this analysis (Supplementary material online, Figure S4). Control-CMs had higher rate of spontaneous beating than DMD- $\Delta$ Exon50- and c.263delG-CMs ( $58.5 \pm 10.43$ vs. $33.43 \pm 4.28$ vs. $35.4 \pm 9.60$ b.p.m. respectively, $P<0.05$ by ANOVA) but had a lower number of spontaneous beating cells than both DMD- $\Delta$ Exon 50 - and c.263delG-CMs ( $8.8 \%$ vs. $20.6 \%$ vs. $17.2 \%$, respectively) (Supplementary material online, Figure S4), supporting the idea that the absence of Dp427 may delay maturation of the excitation contraction coupling.

\section{Loss of Dp427 blunts cardiac contractile reserve}

To investigate the developmental state of the $\mathrm{Ca}^{2+}$ handling apparatus and test the contractile reserve of intact DMD-CMs we used a post-rest potentiation protocol. ${ }^{23}$ In adult cardiac muscle, a resting period introduced during steady-state stimulated pacing enhances $\mathrm{Ca}^{2+}$ release from the sarcoplasmic reticulum (SR) and potentiates contraction from the first post-rest stimulation by multiple mechanisms. This includes increased SR $\mathrm{Ca}^{2+}$ refilling and enhanced RyR2 channel availability, as a consequence of recovery from inactivation/adaptation during the prolonged rest period. Rest periods of $5 \mathrm{~s}$ were introduced during $1 \mathrm{~Hz}$ stimulation of control-, DMD-, and c.263delG-CMs, and the amplitude of the 
Table I Summary of mechanical and kinetic properties of myofibrils from hiPSC-CMs following maximal and submaximal $\mathrm{Ca}^{2+}$ activation

\begin{tabular}{|c|c|c|c|c|c|}
\hline Myofibril type ${ }^{a}$ & $\begin{array}{l}\text { Tension }^{d} \\
\mathrm{mN} / \mathrm{mm}^{2}\end{array}$ & $\begin{array}{l}k_{A C T} \\
s^{-1}\end{array}$ & $\begin{array}{l}k_{\text {REL}}, \text { slow }^{f} \\
s^{-1}\end{array}$ & $\begin{array}{l}t_{\text {REL, }} \text { slow } \\
\text { ms }\end{array}$ & $\begin{array}{l}k_{\text {REL }}, \text { fast }^{h} \\
s^{-1}\end{array}$ \\
\hline \multicolumn{6}{|l|}{$\mathrm{pCa} 4.0^{\mathrm{b}}$} \\
\hline Control (n) & $18.6 \pm 2.5(26)$ & $0.51 \pm 0.04$ & $0.30 \pm 0.04(25)$ & $192 \pm 7.4(29)$ & $2.66 \pm 0.3(25)$ \\
\hline DMD (n) & $5.1 \pm 0.5(23)^{* *}$ & $0.61 \pm 0.09(23)$ & $0.32 \pm 0.04(26)$ & $265 \pm 10(26)^{* *}$ & $1.51 \pm 0.2(26)^{* *}$ \\
\hline \multicolumn{6}{|l|}{ pCa $5.8^{c}$} \\
\hline Control (n) & $8.5 \pm 1.4(22)$ & $0.39 \pm 0.06(22)$ & $0.30 \pm 0.03(17)$ & $154 \pm 7.9(21)$ & $2.47 \pm 0.4(22)$ \\
\hline DMD (n) & $4.0 \pm 0.7(17)^{*}$ & $0.34 \pm 0.05(17)$ & $0.37 \pm 0.06(14)$ & $265 \pm 15(14)^{* *}$ & $1.09 \pm 0.2(15)^{* *}$ \\
\hline $\begin{array}{l}{ }^{\mathrm{a}} \text { Data are mean } \pm \\
\text { each cell line. Numb } \\
\text { b Maximal activating } \\
\text { cSubmaximal activat } \\
{ }^{\mathrm{d}} \text { Tension, maximal, } \\
{ }^{\mathrm{e}} \text { Rate of force gener } \\
{ }^{\mathrm{f}} \text { Slow, rate of the slc } \\
{ }^{\mathrm{g} S} \text { Slow, duration of } \mathrm{t} \\
{ }^{\mathrm{h}} \text { Fast, rate of the fas } \\
* P<0.05 \text {. } \\
* * P<0.01 \text {; estimate }\end{array}$ & $\begin{array}{l}\text { imal } \mathrm{Ca}^{2+} \text {-activated } \\
\text { ving } \mathrm{Ca}^{2+} \text {-activation. } \\
\text { c phase of relaxation } \\
\text { netric phase of relax } \\
\text { elaxation estimated } \mathrm{f} \\
\text { dent's } t \text {-test. }\end{array}$ & $\begin{array}{l}\text { rom the normalized } \\
\text { ing sudden } \mathrm{Ca}^{2+} \text { rem } \\
\text { e constant of the exp }\end{array}$ & $\begin{array}{l}\text { linear fit to the forc } \\
\text { to the force trace. }\end{array}$ & was used and refers & th work. ${ }^{14} N=3$ for \\
\hline
\end{tabular}

post-rest potentiated contraction was plotted against that of the last regular contraction during pacing (Figure 5E). The relative amplitude of post-rest twitches $\left(p_{1} / p_{2}\right)$ was $\sim 25 \%$ less potentiated for DMD- and c.263delG-CMs compared to control-CMs ( $P<0.05$ for both cell lines), suggesting depressed contractile reserve that could result from $\mathrm{Ca}^{2+}$ handling abnormalities (Figure 5F).

\section{Loss of Dp427 alters cardiac $\mathrm{Ca}^{2+}$ transient dynamics}

We analysed intracellular $\mathrm{Ca}^{2+}$ transients of DMD- and c.263delG-CMs (Figure 6) as these, together with myofibril function, determine the contractile properties of cardiomyocytes. For these measurements it was necessary to plate both control- and DMD-CMs on flat gelatine-coated surfaces for simultaneous measures of $\mathrm{Ca}^{2+}$ transients and cell shortening, as the grooves in the nanopatterned surfaces produced light deflection phenomena that interfered with the fluorescent dye signal in our system. Cells were loaded with Fura-2 AM and the fluorescent signal that monitors intracellular $\mathrm{Ca}^{2+}$ was detected through excitation at $510 \mathrm{~nm}$ wavelength using a fluorescence detection system (lonOptix) mounted on an inverted microscope. Representative traces are shown in Figure 6A, B. There were no significant differences of baseline (resting) $\mathrm{Ca}^{2+}$ or the maximal $\mathrm{Ca}^{2+}$ transient amplitude (Figure $6 D-F$ ), though there did appear to be more variability in transient amplitudes for DMDCMs (Figure 6E). However, the kinetics of $\mathrm{Ca}^{2+}$ transients were significantly impaired in DMD-CMs (Figure 5C). The transient rise from baseline to $50 \%$ of the signal peak was similar for most stimulation protocols, except for $0.5 \mathrm{~Hz}$ for DMD-CMs, where it was significantly slower $(P<0.01)$. The time to the peak of the $\mathrm{Ca}^{2+}$ transient was significantly prolonged for DMD-CMs, but not for c.263delG-CMs, at all stimulation frequencies (Figure 6A,C). As a result, the shape of DMD-CM intracellular $\mathrm{Ca}^{2+}$ transient rise at $0.5 \mathrm{~Hz}$ stimulation was biphasic, with an initial rapid rise, followed by a slower phase to reach the peak (Figure 6A). While differences in the rise of intracellular $\mathrm{Ca}^{2+}$ appeared to be minimal, the decay of $\mathrm{Ca}^{2+}$ transients was greatly prolonged for both DMD-CMs and c.263delG-CMs. The half time of $\mathrm{Ca}^{2+}$ transients decay (RT50; measured as time from peak to $50 \% \mathrm{Ca}^{2+}$ transient decay) was considerably longer compared to control-CM at all pacing frequencies (Figure 6C). Taken together, the combined results indicate that both cellular $\mathrm{Ca}^{2+}$ handling and myofibril contractile abnormalities underlie the difference in contractile properties seen in the DMD cell lines.

\section{Dystrophin domains and costamere formation in control cells}

Finally, we investigated whether functional differences are associated with differences in costamere formation and alignment with Z-bands that occurs in normal cardiomyocyte development. Caveolin 3 (Cav3) is a scaffolding protein that interacts with the dystrophin-associated glycoprotein complex (DGC) and is involved in T-tubule formation in the striated muscle. ${ }^{24}$ We co-stained control- and DMD-CMs for Z-bands (anti $\alpha$-actinin) and caveolin 3 (anti-Cav3). DMD-CMs had negligible Cav3 localization within the sarcolemma (Supplementary material online, Figure S6A) compared to control-CMs (control $r_{\text {coloc }}=0.37 \pm 0.05$ vs. DMD $r_{\text {coloc }}=0.05 \pm 0.04, P<0.01$ ) (Supplementary material online, Figure S6B). Cav3 domains, identified with the help of 3D image reconstruction, were rarely observed in DMD-CMs. This may suggest reduced ability for caveolae/T-tubule formation in DMD-CMs, though this needs to be investigated further. We also used immunohistochemistry to evaluate dystrophin distribution (anti-dystrophin) and its association with Z-bands (anti- $\alpha$-actinin) of hiPSC-CMs cultured on nanopatterned surfaces (Supplementary material online, Figure S7A, B). At 40 days post-differentiation, 3D confocal images reconstruction of control-CMs displayed a high level of dystrophin expression, forming an extensive network throughout the cell volume (Figure 7A). No detectable Dp427 was observed in DMD- $\Delta$ Exon50- or c.263delG-CMs (Figures S2 and S3). Figure $7 B$ shows the transverse plane (bottom view) and the sagittal plane (3Dlateral view) of a 3D-control-CM (maximal cell height was $16.11 \pm 1.25 \mu \mathrm{m})$. For the cell membrane close to the nanopatterned surface (bottom view), there was a transverse pattern of dystrophin that formed clusters following the alignment of the nanogrooved substrate along cell edges (Figure 7B, second panel). Throughout the entire cell surface, aligned myofibrils were highly co-localized with dystrophin $\left(\mathrm{r}_{\text {coloc }}=\right.$ $0.358 \pm 0.02$ ). Moreover, along the cell edges lateral distribution of dystrophin showed more marked signs of dystrophin clusters that tightly juxtaposed Z-band domains with matching periodicity (dystrophin to 
A
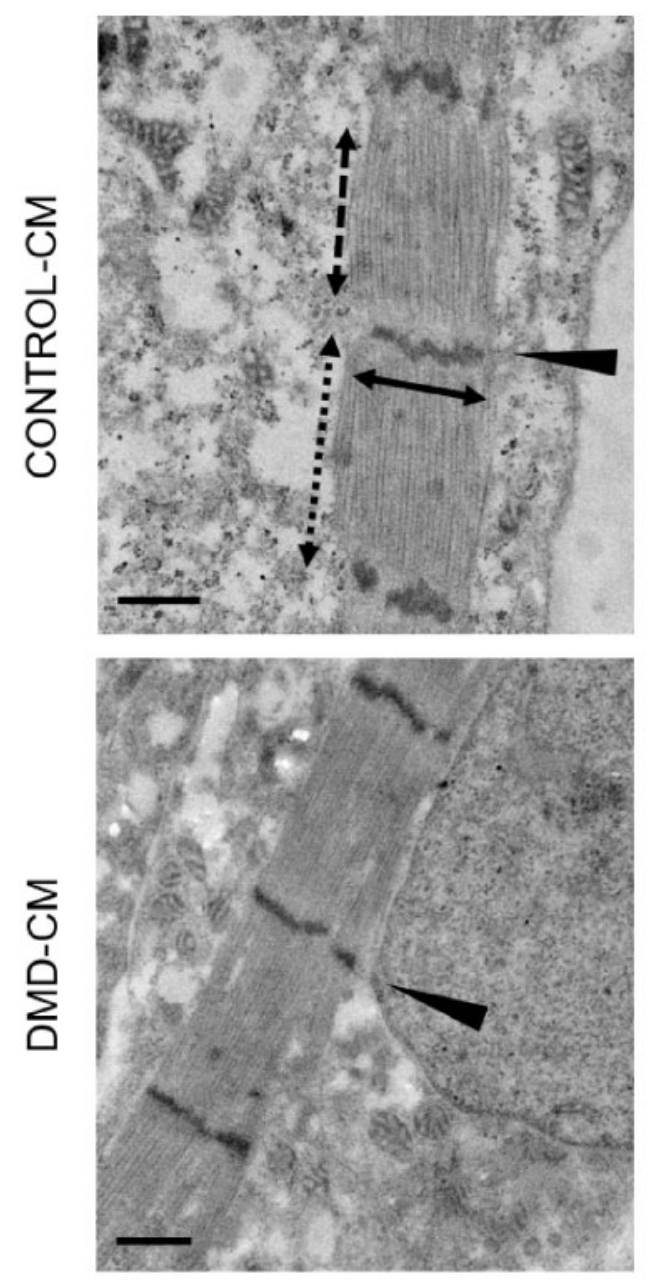

B
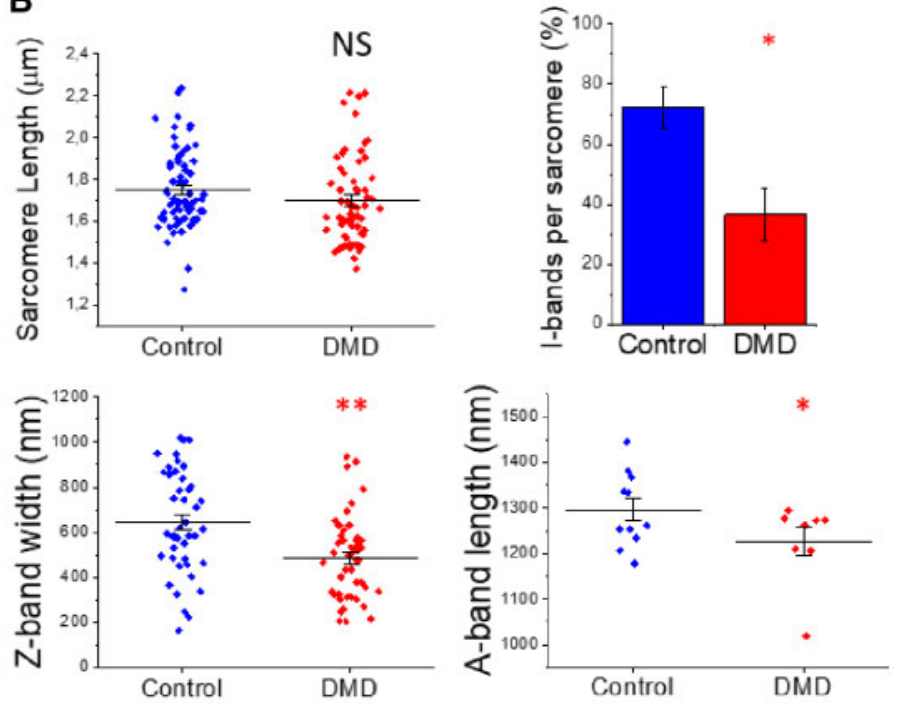

C

a-MHC

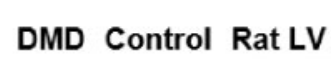

kDa
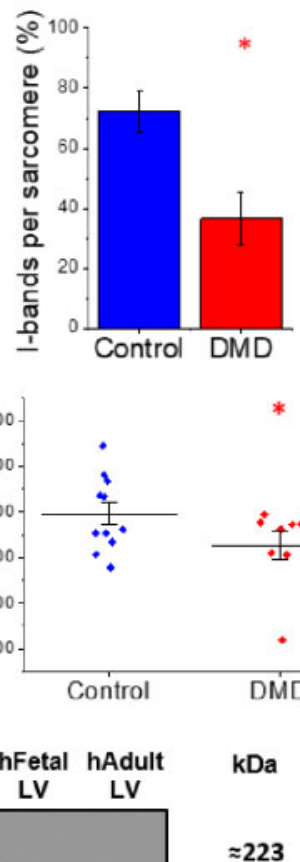

$\beta-\mathrm{MHC}$

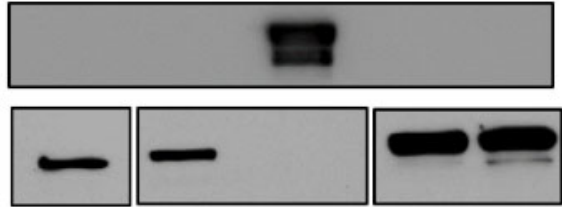

$\approx 223$

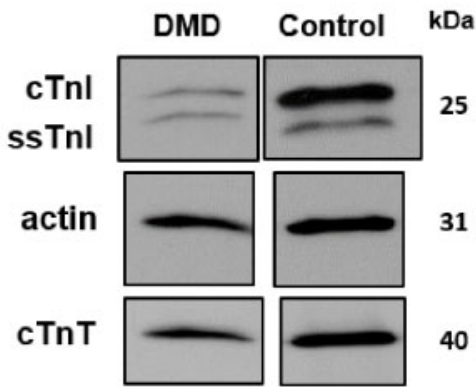

Figure 4 Sarcomere composition of DMD-hiPSC-CMs. Single DMD-(hiPSC)-CMs sparsely cultured on nanopatterned surfaces at 100 days post differentiation. (A) Transmission electron microscopy (TEM) at high magnification (40 000 $\times$ ) showed aligned Z-bands (black arrowheads). (B) Observations of DMD resting sarcomere length (SL; black dotted two-headed arrow), I-bands per sarcomere (\%), Z-band width (cross sectional length; black solid twoheaded arrow) and A-band length (black dashed two-headed arrow). (C) Sarcomere protein expression of DMD-CMs were compared to control-CMs, foetal left ventricular (LV) samples (130 gestational days) and adult human and rat LV. The lower panels demonstrate detection of $\alpha$ - and $\beta-M H C$ (top), cTnl, and ssTnl from a single gel. Single panels are cropped image from the same gels. Uncropped images are shown in Supplementary material online, Figure S6. $N=3$ for each cell line, $n$ of sarcomeres was 56-62 per cell line for a total of three individual experiments. Scale bars equal to $500 \mathrm{~nm}$. Average are reported as means $\pm \mathrm{SEM} ; * * P<0.01$; $* P<0.05$; NS, not significant; estimated by one-way ANOVA with Tukey post hoc test.

dystrophin, $\mathrm{DD}_{\text {length }}=1.91 \pm 0.05 \mu \mathrm{m}$ vs. $\mathrm{SL}=1.89 \pm 0.04 \mu \mathrm{m}, P=0.74$ ), suggesting increasing organization and stabilization of costameric connections to myofibrils (Figure 7B, Supplementary material online, Figure S7).

\section{Discussion}

Cardiac complications are a leading cause of morbidity and mortality in DMD patients. Studies in animal models have provided some understanding, as they recreate some aspects of the human cardiac phenotype. ${ }^{25}$ However, human dystrophin deficiency that leads to DMD cardiomyopathy needs to be modelled to validate pathogenic mechanisms and to identify disease-specific molecular targets that may be amenable to therapeutic intervention. Patient-derived and CRISPR-Cas9 edited hiPSC-CMs offer a cell level model to study the early-stages of human DMD cardiomyopathy, as opposed to the latter stages of the disease. Our stem cell to cardiomyocyte differentiation and culturing protocol may recapitulate critical developmental steps in the cardiac lineage, thus allowing us to observe the first molecular, structural, and functional consequences downstream of full-length dystrophin (Dp427m) deficiency (Figure 8). A single base in exon 1 in DMD gene was deleted 
A
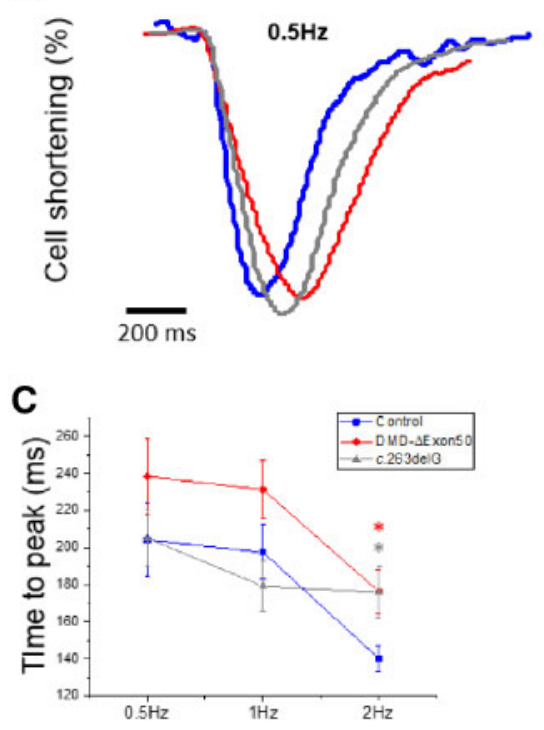

E

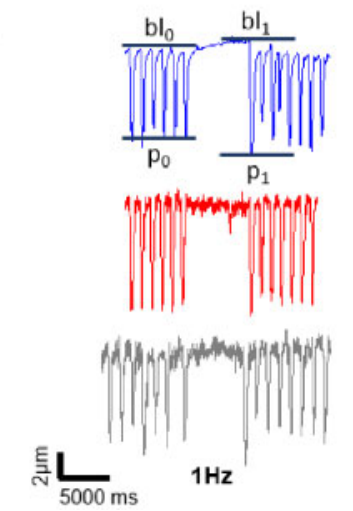

B
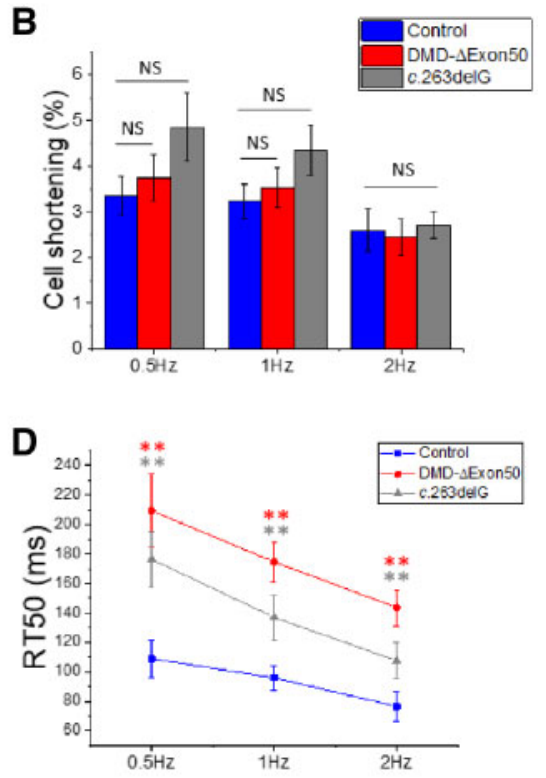

$\mathbf{F}$

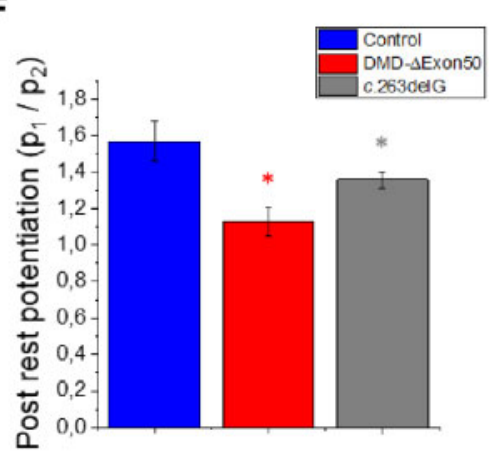

Figure 5 Intact cell contractility of DMD-CMs. Intact cell contractility was measured on flat surfaces (80 days p.d.) for simultaneous detection of $\mathrm{Ca}^{2+}$ transients (Figure 6) at $37^{\circ} \mathrm{C}$ and $1.8 \mathrm{mM}$ external $\mathrm{Ca}^{2+}$. DMD-CMs were compared with control- and c.263delG-CMs at three different pacing frequencies $(0.5,1,2 \mathrm{~Hz})$. (A) Representative traces of control-, DMD-, and c.263delG-CMs showed (B) preserved cell contractility (\% of fractional shortening) at any pacing frequency, $(C)$ slower time to peak of contraction at $2 \mathrm{~Hz}$, and $(D)$ slower relaxation kinetic (RT50\%) at all frequencies. For cell contractility analysis $N=5$ for control and DMD and $N=3$ for c.263delG, $n=18-20$ for each cell line for a total of five individual experiments. (E) A post-rest potentiation protocol was employed at $1 \mathrm{~Hz}$, with a resting pause of $5 \mathrm{~s}$ in each cell line. The potentiation is estimated by $\mathrm{p}_{1} / \mathrm{p}_{0}$ (peak amplitude post-rest/before rest), bl, baseline. (F) Both DMD- and c.263delG-CMs had depressed potentiation of contraction ( $n=11-13$ per cell line); $n$ is the number of cells. Average are reported as means $\pm \mathrm{SEM} ; * * P<0.01 ; * P<0.05$; NS, not significant; estimated by two-way ANOVA with Tukey post hoc test.

(c.263delG) and Dp427m was not detected in the isogenic cardiomyocytes. We found expression of a truncated dystrophin, likely due to the presence of a promoter in exon 6. However, our results demonstrate that c.263delG cardiomyocytes mirror DMD patient cardiomyocytes (Supplementary material online, Table S2), strongly suggesting that the truncated dystrophin lacking of the $\mathrm{N}$-terminal actin-binding domain (Exons 1-5) does not provide stability as the Dp427. This demonstrated that absence of full-length dystrophin alone is sufficient to cause development of dysfunctional differences that are likely a factor in development of the cardiac disease phenotype. We have provided an in depth analysis of isolated myofibril contractile mechanics and kinetics and the $\mathrm{Ca}^{2+}$ handling properties of DMD-CMs, and identified structural and protein correlates that may be responsible for differences from control-CMs.
The morphology of DMD- and control hiPSC-CMs (Figure 2) in this study was matured (cultured for 80-100 days) far beyond previous reports for the same cell lines, which were grown on nanopatterned surfaces for less than 35 days. ${ }^{13,15}$ Longer culturing in the current study has significant advantages for the identification of biophysical alterations that occur early in development and dissection of the pathophysiological mechanisms associated with the disease. DMD-CMs in this study displayed prominent cell hypertrophy that could be a normal outcome of the maturation process, but also may be a compensatory mechanism in response to impaired contractile properties, as discussed below. This also occurred with less mature DMD-CMs (of the same cell line) grown on the same nanopatterned surfaces, ${ }^{13}$ but longer culturing resulted in much larger cells and a greater hypertrophy in DMD compared with control cells. Importantly, both DMD and control cell lines displayed 


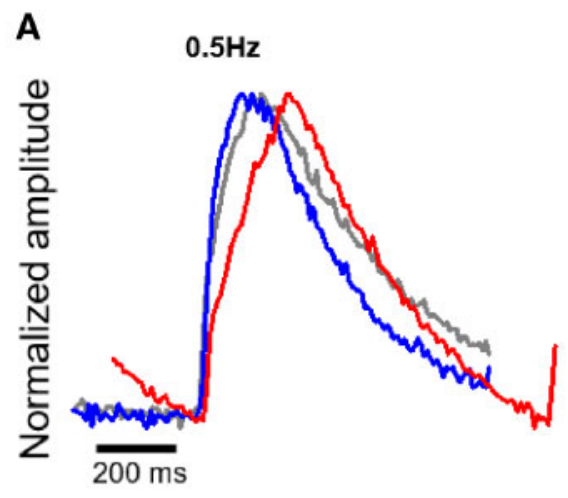

B
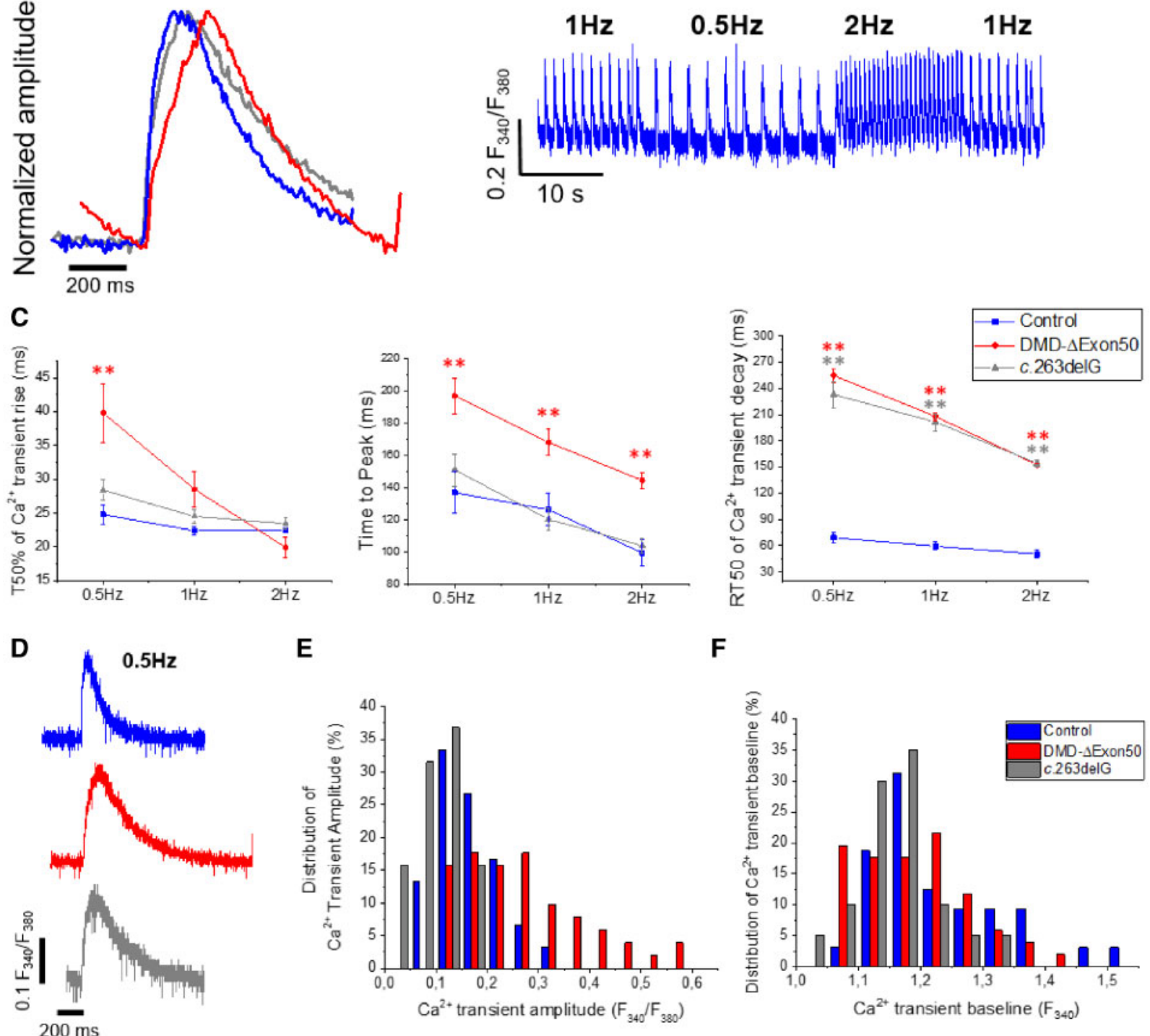

Figure $6 \mathrm{Ca}^{2+}$ handling abnormalities of DMD-CMs. Ca ${ }^{2+}$ transient measurements of control-, DMD- $\Delta$ Exon50 and c.263delG-CMs detected by Fura2AM fluorescent dye. (A) Representative traces $(0.5 \mathrm{~Hz})$ were normalized to show $\mathrm{Ca}^{2+}$ transient kinetics. (B) Control- (representative), DMD- and c.263delG-CMs were paced at three different frequencies $(0.5,1,2 \mathrm{~Hz})$. (C) Slower $\mathrm{Ca}^{2+}$ transient decay of DMD- and c.263delG-CMs at any frequency. (D) Representative $\mathrm{Ca}^{2+}$ transients of control-, DMD- and c.263delG-CMs $(0.5 \mathrm{~Hz})$. (E) The frequency count for DMD- $\Delta$ Exon 50 exhibited variable Ca ${ }^{2+}$ transient amplitudes $\left(\mathrm{F}_{340} / \mathrm{F}_{380}\right)$ within the population, while c.263delG had a Gaussian distribution similarly to control-CMs. ( $\left.F\right)$ Similar distribution of $\mathrm{Ca}^{2+}$ transient baseline. Both distributions are shown at $0.5 \mathrm{~Hz}$ but were also variable at the other frequencies (data not shown). The distribution of the values in the frequency count were confident within the cell lines. For $\mathrm{Ca}^{2+}$ transient analysis $N=5$ for control and DMD and $N=3$ for $c .263$ delG, $n=37-52$ per cell line for a total of five individual experiments. Average are reported as means \pm SEM; $* * P<0.01 ; * P<0.05$; NS, not significant; estimated by two-way ANOVA with Tukey post hoc test.

similar myofibril alignment and resting sarcomere length. This result contrasts with previous findings for less mature DMD-CMs and a hiPSC-CM cell line carrying a dilated cardiomyopathy-associated mutation ${ }^{13,26}$ on flat surfaces, both of which impair myofibril alignment in the diseased cells. This suggests in absence of Dp427, myofibrils may be less able to organize or bundle in the absence of strong external, topographical cues and are more susceptible to disorganization under stress. It also supports the idea that disease-causing mutations can precipitate physiological defects early in cardiac lineage commitment, but some aspects of the disease pathology require further maturation and/or in vitro aging for full manifestation of the disease phenotype.

To establish a linkage between dystrophin and sarcomeres, both dystrophin and $\alpha$-actinin (Z-bands) were labelled in control-hiPSC-CMs. We confirmed that early in post-differentiated hiPSC-CMs, dystrophin is uniformly distributed in a wide network. At cell edges dystrophin domains appeared in line with Z-bands with matching periodicity. This is 
A
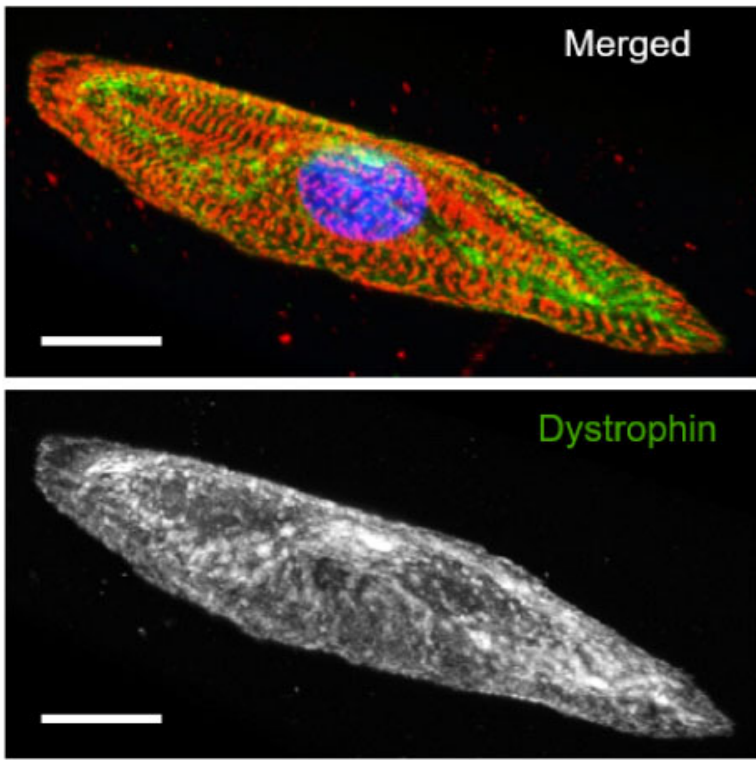

B

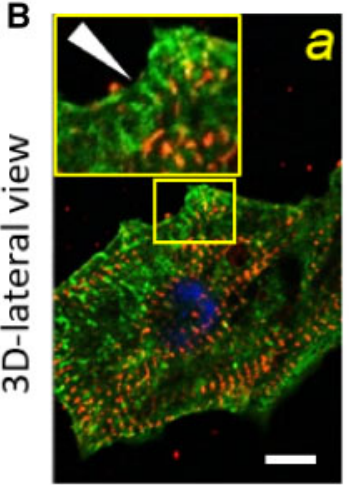

Bottom view
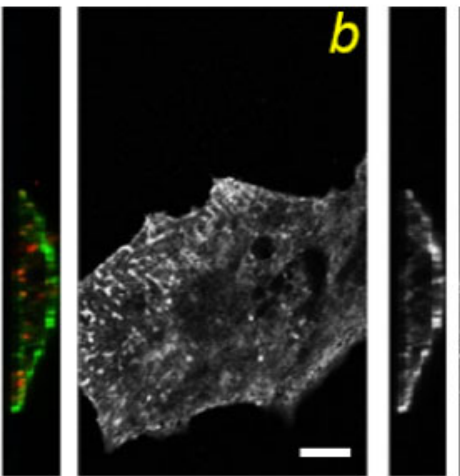
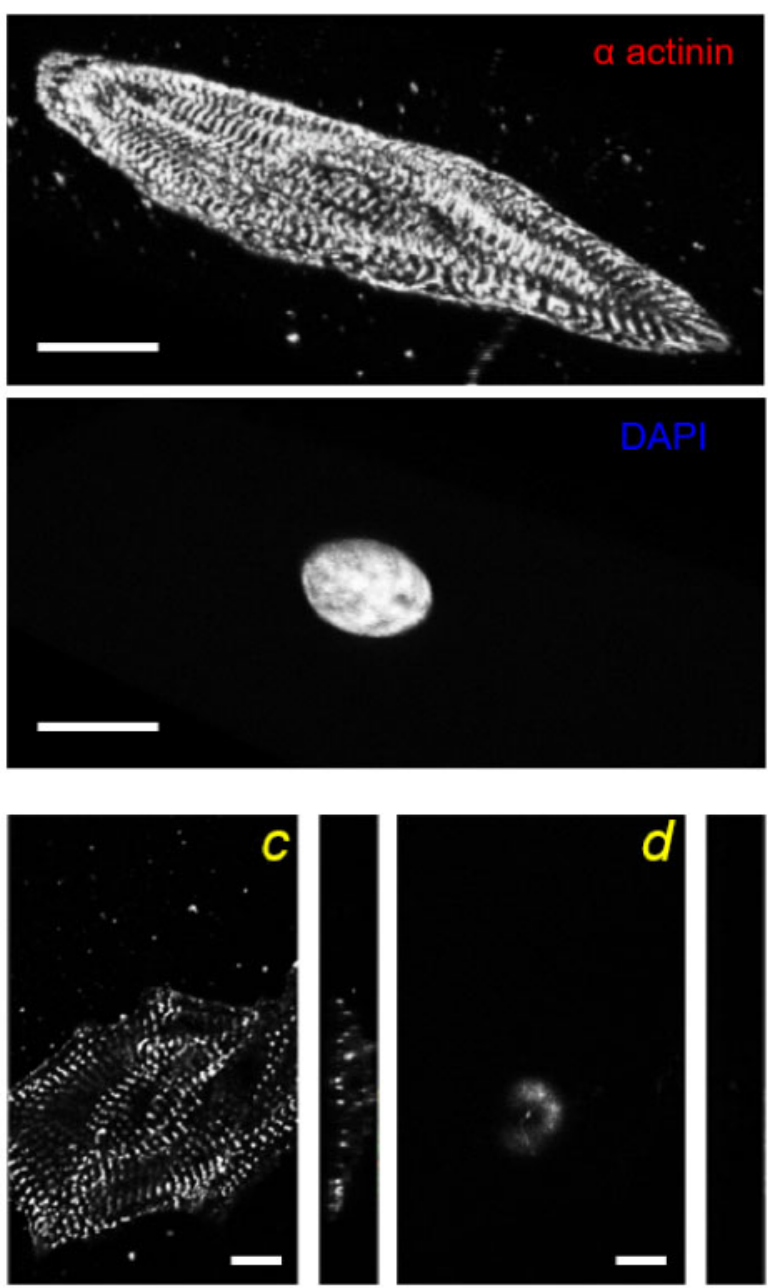

Figure 7 Myofibril alignment and dystrophin network during nanopatterned-mediated maturation. Confocal images of control-(hiPSC)-CMs cultured on the nanogrooved substrates. (A) Control-CMs were stained with antibodies against Z-bands ( $\alpha$-actinin, red), dystrophin (green) and nuclei (DAPI, blue). Representative 3D reconstructions showed clear myofibril alignment quantified by fast Fourier transform (FFT) analysis. Dystrophin network formation occurred early (40 p.d. days) and covered the entire sarcolemma. (B) The bottom view of a hiPSC-CM showed dystrophin distribution (green) in a bi-dimensional view (a). The yellow-outlined blow-up shows an area of cell edge with dystrophin clusters and the same periodicity of Z-bands (a). Black and white panels show single staining of dystrophin (b), $\alpha$-actinin (c), and DAPI (d). Dystrophin domains juxtaposed $\alpha$-actinin staining (white arrowhead, left panel). For this analysis, the number of 3D-cell reconstruction was 12 . Scale bars equal to $20 \mu \mathrm{m}$. One-way ANOVA with Tukey post hoc test.

similar to previous observations in wild-type mice, ${ }^{27}$ suggesting codependence in forming costameric regions. These findings also support the idea that costameres are hotspots during early stages of hiPSC-CM maturation, similar to human foetal ventricular tissue. ${ }^{7}$ Since myofibrils were aligned in both control and DMD-cell lines, it also suggests that reduction or loss of costamere formation may affect the development of the contractile apparatus.

Our custom-built apparatus for isolated myofibril preparations allows evaluation of isolated myofibril mechanics and kinetics of activation and relaxation, independent of the $\mathrm{Ca}^{2+}$ handling system of hiPSC-CMs. ${ }^{14}$ We were initially surprised to find much weaker myofibril tension generating capacity for myofibrils from dystrophin-deficient CMs, as dystrophin is not a sarcomere protein. However, the kinetics of tension development $\left(k_{\mathrm{ACT}}\right)$ and the early phase of relaxation (slow $\left.k_{\mathrm{REL}}\right)$, which reflect the properties of the isovolumetric phase of systole and early phase diastole in hearts (respectively), were not different compared to myofibrils from control-CMs. This suggests similar acto-myosin cycling kinetics that is likely reflective of the predominant expression of the $\beta-M H C$ isoform in both DMD- and control-CMs at this stage of maturation. It also parallels what we found for developing foetal ventricular myocardium, where the switch from $\alpha$ - to $\beta-M H C$ isoform occurs very early (within two months) in human ventricular myocardial development. ${ }^{9,10}$ Our finding that average Z-band diameters of individual myofibrils were significantly smaller for DMD-CMs suggests myofibril assembly may be impacted by the lack of full-length dystrophin. It is possible that defects in the mechano-transduction signals between DGC and Z-bands could result from a lack of Dp427m, and this may interfere with correct myofibril formation and organization. ${ }^{3}$ Interacting proteins 


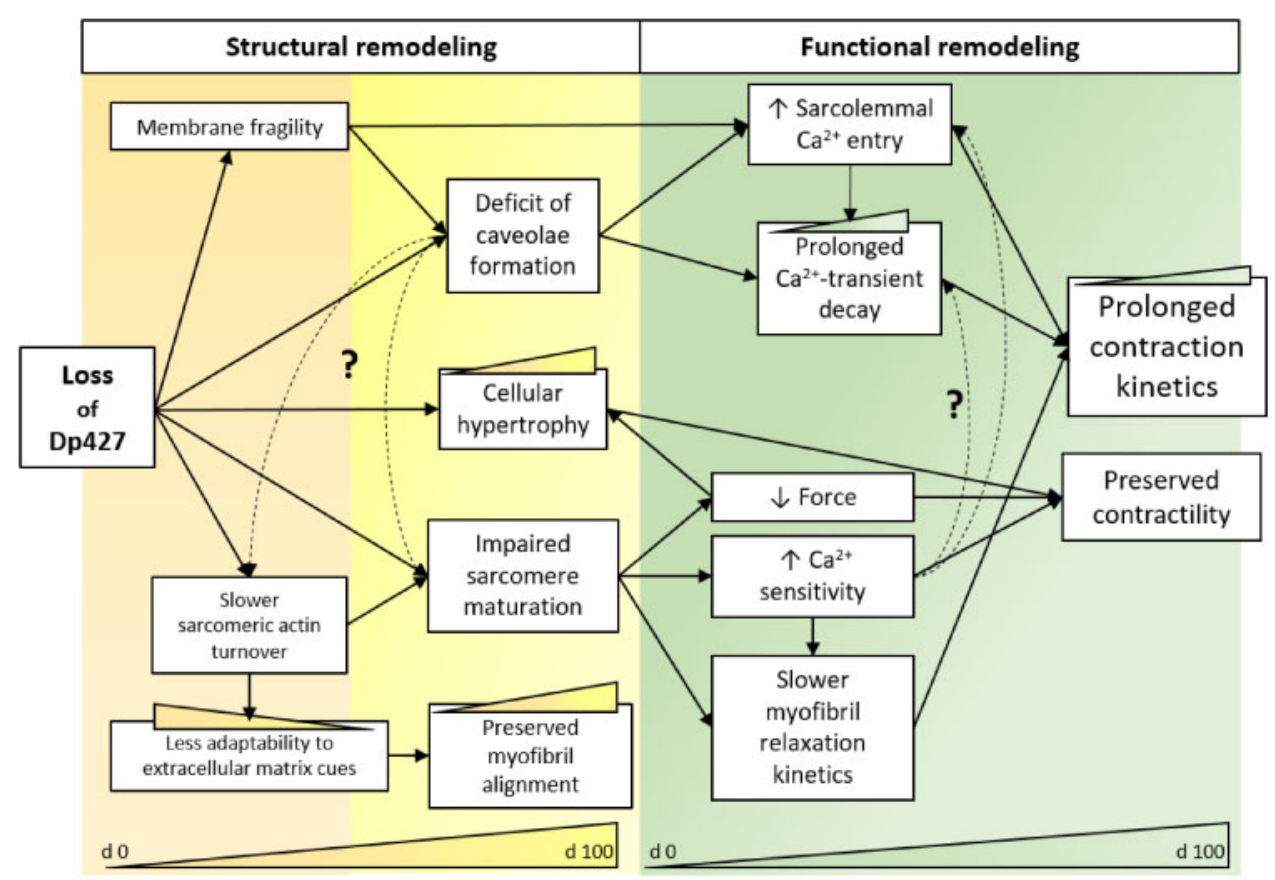

Figure 8 Schematic representation of loss of Dp427 consequences in hiPSC-CMs. Consequences of the altered genotype (loss of Dp427) on phenotypic adaptions include structural-functional alterations in DMD-hiPSC-CMs (orange/yellow-green panel respectively). Deficit of caveoloe formation may slow down $\mathrm{Ca}^{2+}$ transients and maintain more dependence to sarcolemmal $\mathrm{Ca}^{2+}$ entry. On the other side, impaired maturation of specific sarcomere determinants may result in weaker myofibril tension production. Increased $\mathrm{Ca}^{2+}$-sensitivity of tension development and cellular hypertrophy may potentially rescue cell contractility at low load. Slower $\mathrm{Ca}^{2+}$ transient kinetics and myofibril relaxation are likely a main cause of slower cell contractility. Dashed lines refer to hypothetic mechanisms to explore. Colourful progress bars (Days 0 to 100 from cardiac differentiation) refer to findings of this work and previous studies on these DMD-hiPSC-CM lines. ${ }^{13,15}$

of costameres/Z-bands are in a well-suited location to influence correct myofibrillogenesis. ${ }^{12}$ The lower tension in DMD-CM myofibrils is most easily explained by delayed or abnormal maturation of sarcomere ultrastructure (Figure 3B) or by reduced myosin density within the sarcomere that could reduce cross-bridge binding during contraction. In support of this idea, previous studies with DMD-hiPSC-CMs report a reduced transcription profile of sarcomere genes ${ }^{28}$ and reduced sarcomere actin turnover. ${ }^{13}$ Therefore, the hypertrophic response in DMD-hiPSC-CMs reported in this and our previous study ${ }^{13}$ may well be a mechanism to compensate for weaker tension generating capacity of individual myofibrils. Of note, external environmental conditions (i.e. nanotopography) may play a major role in exacerbating the DMD-hiPSC-CM phenotype, but cell morphology may adapt differently in vivo during DMD cardiomyopathy progression.

Another potential compensatory mechanism is the greater $\mathrm{Ca}^{2+}$ sensitivity ( $\mathrm{PC} \mathrm{a}_{50}$ ) of tension development for myofibrils from DMD-hiPSC$\mathrm{CMs}$, which could result from differences in thin filament protein isoform expression or phosphorylation patterns. An in depth proteomic analysis was beyond the scope of our current study, due to the low density of cells on nanopatterned surfaces (by design, to ensure individual cells) and the length of the culture period (80-100 days). Because of this, we felt it was important to focus on contractile properties for this report, and this limited the amount of contractile material available for protein analysis. The prolonged relaxation of myofibrils from DMD-hiPSC-CMs, even though myosin isoform did not differ from control hiPSC-CM myofibrils, may indicate slower $\mathrm{Ca}^{2+}$ dissociation from troponin in
DMD-CM myofibrils, an idea supported by the increased $\mathrm{pCa}_{50}$ (myofibril Ca ${ }^{2+}$ sensitivity). ${ }^{22,29}$ Indeed, we have demonstrated that slower troponin kofF can slow myofibril relaxation, prolonging the early, slow phase of relaxation $\left(t_{\mathrm{REL}}\right)$ and the larger, fast component of relaxation (fast $\left.k_{\text {REL }}\right) .^{22}$ The small amount of protein available following myofibril mechanical experiments was not sufficient to establish whether there were differences in the amount of the adult cardiac form of troponin I (cTnl) vs. the embryonic isoform slow skeletal (ssTnl) incorporated into myofibrils, or other post-translational modifications (e.g. phosphorylations), both of which could impact the $\mathrm{Ca}^{2+}$ binding properties of troponin. ${ }^{10,30}$ However, our ultrastructural analysis suggests that lack of Dp427 may influence the rate of sarcomere structure maturation even if protein isoform expression patterns are similar, and this may compromise the contractile properties of single myofibrils.

Another limitation of this work was the inability to measure $\mathrm{Ca}^{2+}$ transient behaviour from cells on nanopatterns, due to the reflective properties of the grooves in the surface. This necessitated measuring $\mathrm{Ca}^{2+}$ transient behaviour under a different culture condition (flat matrix surfaces). In previous experiments myofibrils from hiPSC-CMs cells cultured on gelatin-coated surfaces were less aligned and dense, ${ }^{13}$ though we have demonstrated that long-term culture can reduce this shortcoming. ${ }^{31}$ Interestingly, the magnitude of contraction (cell fractional shortening) of DMD- $\Delta$ Exon50- and c.263delG-CMs was similar to controls. This may be due in part to the apparent increase in myofibril $\mathrm{Ca}^{2+}$ sensitivity and/or longer $\mathrm{Ca}^{2+}$ transient duration. Additionally, measurements of contraction in culture are done at low load, which can mask contractile 
deficits associated with reduced tension producing capacity during contraction. An additional possible consequence of the lack of Dp427 during hiPSC-CM maturation in culture may be mitochondrial damage, due to oxidative stress. This has often been reported as a leading factor in the pathogenesis of DMD, and early-stage DMD-hiPSC-CMs from the patient with exon 50 deletion had faster mitochondrial permeability pore open, ${ }^{15}$ which was confirmed in a study by Lin and colleagues. ${ }^{28}$ Altered mitochondrial ATP production may influence the energy available for contraction and $\mathrm{Ca}^{2+}$ cycling, affecting SERCA pump activity that restores $\mathrm{Ca}^{2+}$ to the SR. In addition, nitric oxide synthase (NOS)-induced ROS and subsequent genomic instability were reported to be close connected to loss of dystrophin in hiPSCs. ${ }^{32}$ Oxidative stress may also cause irreversible post-translational modifications on myofibril formation and/or function via carboxylation, glutathionylation or nitrosylation of amino acid residues. This may have contributed to lower force production in DMD myofibrils and perhaps the prolongation of the early, slow phase of relaxation ( $t_{\mathrm{REL}, \text { slow }}$ ) that is modulated by thin filament deactivation rates. 22

In support of these findings, twitch amplitude was blunted under inotropic intervention, measured as post-rest contractions, due to reduced contractile reserve in DMD-CMs. This can be explained by $\mathrm{Ca}^{2+}$ handling abnormalities, possibly related to alteration of $\mathrm{SR}$ regulation, maturation or volume. Altered $\mathrm{Ca}^{2+}$ handling is considered a hallmark due to loss of Dp427 in several models. ${ }^{2}$ DMD-CMs have slower $\mathrm{Ca}^{2+}$ transient decay in the early stage post differentiation ${ }^{15}$ and here we found that this increased as DMD-CMs age. The difference in RT50 of $\mathrm{Ca}^{2+}$ transient decay could result from slower $\mathrm{Ca}^{2+}$ refilling on the SR by SERCA pumps and/or slower $\mathrm{Ca}^{2+}$ extrusion from the $\mathrm{Na}^{2+} / \mathrm{Ca}^{2+}$ exchanger (NCX). Studies in $m d x$ mice suggest a myriad of mechanisms for altered $\mathrm{Ca}^{2+}$ handling in cardiac muscle, ${ }^{2,33}$ thus future studies should explore pathways regulating the $\mathrm{Ca}^{2+}$ handling abnormalities of DMDCMs. For instance, within DGC-associated proteins, Cav3 is a scaffolding protein localized at the opening of caveolae and $\mathrm{T}$ tubules, responsible for their formation and provides stability to the muscle cell membrane. ${ }^{34,35}$ Not least, Cav3 upregulation progressively clusters with $\mathrm{HCN} 4$ ( $\mathrm{l}_{\mathrm{f}}$ current) and is correlated by decreased spontaneous beating rate in later stages post differentiation of hESC-CMs. ${ }^{36,37}$ As reported for adult $m d x$ mice $^{38}$ a deficit or delayed formation of T-tubules in DMD-CMs may be a specific disorder of DMD cardiomyopathy, since we previously observed a relatively strong presence of Cav3 profiles in a hiPSC-CM model of familial cardiomyopathy that have similar deficits in myofibril contraction strength. ${ }^{14}$ Absence of Dp427 may lead to aberrant caveolae or T-tubule-like architecture and alter the signalling pathways of costameric proteins, limiting the maturation process of single sarcomeres. However, the current study provided only cursory evidence for this, and further studies are needed to draw any conclusions regarding this mechanism.

\section{Translational implications and study limitations}

It has long been thought that clinical manifestations of DMD cardiomyopathy were predominantly a secondary effect due to a state of deterioration that progresses as DMD patients age. ${ }^{39}$ Despite pathogenesis being apparent during foetal striated muscle cell development ${ }^{40}$ and similar mechanisms that are primarily related to $\mathrm{Ca}^{2+}$ homeostasis, ${ }^{25}$ in vivo studies on $m d x$ mice suggested that the cardiomyopathy may be independent of skeletal muscle disease. ${ }^{41}$ While there may certainly be some variance in cell lines derived from different DMD patients, due to epigenetic and other factors, use of single gene editing to create an isogenic pair of full-length dystrophin knockout and control cell lines is a rigorous means of testing the role of an individual protein. ${ }^{42}$ However, a complete dystrophin knockout or more cell lines with similar exon mutations would be required for creating and studying DMD. In the present work, the similar results between the patient and the CRISPR-Cas9edited cell lines provide direct evidence that the lack of Dp427 directly affects cardiomyocyte function, and the cardiomyopathy is not a secondary remodelling due to systemic alterations (i.e. airway obstruction). Our findings demonstrate that Dp427 deficiency results in delayed or abnormal maturation of cardiomyocyte structure and function, which may resemble some developmental aspects of DMD cardiomyopathy that manifest in the young human adult. ${ }^{1,43}$ Echocardiographic findings ${ }^{44}$ and left ventricular tonic contraction (LV TC, or underfilling) ${ }^{45}$ in DMD patients have demonstrated evidence of diastolic dysfunction in the early stages preceding the development of heart failure, systolic ventricular dysfunction, or dilated cardiomyopathy.

We believe that hiPSC-CMs can provide a platform to investigate the earliest stages of disease development. However, while the structure/ function abnormalities found in hiPSC-CMs can recapitulate aspects of the dystrophic cardiomyopathy, these mechanisms may not necessarily appear in the human heart until birth, which is conditioned by different loading conditions (i.e. cardiac hypertrophy). In addition, early respiratory involvement and loss of ambulation may distinctly aggravate the cardiac phenotype during the paediatric age.

DMD cardiomyopathy is still treated with the classical therapy for heart failure ${ }^{1}$ and clinical observations suggest that a pharmacological prophylaxis can effectively reduce the occurrence of dilated cardiomyopathy within the paediatric age range. ${ }^{45}$ Therefore, the DMD-CM platform presented here could be used as a model to discover and test novel therapies targeted at halting heart disease progression in DMD.

\section{Supplementary material}

Supplementary material is available at Cardiovascular Research online.

\section{Author contributions}

All experiments were conducted at the University of Washington. J.M.P. contributed to the overall study design, performed cardiac differentiation and all experiments, analysed and interpreted data, and wrote the manuscript. D.L.M. designed and with X.G. generated the UC1015-6 cell line. X.G. contributed to confocal microscopy imaging and with D.L.M. and M.K.C. provided control (UC3-4 A1), DMD cell lines (UC72039). J.M.K. performed the western blot analysis. A.W.R. contributed to the myofibril experiments. L.P. provided training assistance on cardiac differentiation. V.M. contributed to confocal microscopy imaging. J.M. and D.-H.K. fabricated the ANFS. M.R.H. and R.L.M. performed mass spectroscopy analysis. C.F., C.T., and C.P. contributed to the overall results interpretation. C.E.M. contributed to the overall study design and provided training assistance. M.R. and D.L.M contributed to the overall study design, interpretation of the results, and writing of the manuscript. All authors critically reviewed the manuscript and approved the final version for publication.

\section{Acknowledgements}

We thank Elisabetta Cerbai for the critical review of the manuscript and Sarah Li for the technical assistance on the proteomic analysis. 


\section{Funding}

M.R. is an established Investigator of the American Heart Association. This work was supported by NIH awards HL111197, HD048895 and HL128368 (M.R.), U01 HL100405, P01 GM081619, R01 HL084642, P01 HL094374 and an award from the Fondation Leducq Transatlantic Network of Excellence (C.E.M.) and a research grant from the Muscular Dystrophy Association to M.K.C., the Senator Paul D. Wellstone Muscular Dystrophy Cooperative Research Center, Seattle (NIH U54AR065139) and by the National Institute of Health R01HL135143 (to D.-H. K.), National Institutes of Health, National Institute of General Medical Sciences under award number R01GM087221 (to R.L.M.), and the National Institutes of Health National Center for Research Resources under award number S10RR027584 (R.L.M.). J.M.P. was supported by a post-doctoral fellowship from a Telethon Italy grant GGP16191 (C.F.). J.M.K. was supported by a Heart and Stroke Foundation of Canada postdoctoral fellowship. A.W.R. was supported by a Marie Sklodowska-Curie fellowship at the University of Kent, UK. X.G. was supported by an American Heart Association fellowship. For the electron microscopy imaging we thank Edward Parker from the Vision Research Center Core at the University of Washington, funded by $\mathrm{NIH}$ award number P30 EY01730.

Conflict of interest: D.-H.K. is a co-founder and scientific advisor of NanoSurface. C.E.M. is the scientific founder of and equity holder in Cytocardia. Other authors have nothing to declare.

\section{References}

1. McNally EM, Kaltman JR, Benson DW, Canter CE, Cripe LH, Duan D, Finder JD, Groh WJ, Hoffman EP, Judge DP, Kertesz N, Kinnett K, Kirsch R, Metzger JM, Pearson GD, Rafael-Fortney JA, Raman SV, Spurney CF, Targum SL, Wagner KR, Markham LW, Working G, Of The NHL, Blood I, Parent P, Muscular D. Contemporary cardiac issues in Duchenne muscular dystrophy. Working Group of the National Heart, Lung, and Blood Institute in collaboration with Parent Project Muscular Dystrophy. Circulation 2015;131:1590-1598.

2. Shirokova N, Niggli E. Cardiac phenotype of Duchenne muscular dystrophy: insights from cellular studies. J Mol Cell Cardiol 2013;58:217-224.

3. Meng H, Leddy J], Frank J, Holland P, Tuana BS. The association of cardiac dystrophin with myofibrils/Z-disc regions in cardiac muscle suggests a novel role in the contractile apparatus. J Biol Chem 1996;271:12364-12371.

4. Kaprielian RR, Stevenson S, Rothery SM, Cullen MJ, Severs NJ. Distinct patterns of dystrophin organization in myocyte sarcolemma and transverse tubules of normal and diseased human myocardium. Circulation 2000;101:2586-2594.

5. Vatta M, Sinagra G, Brunelli L, Faulkner G. Remodeling of dystrophin and sarcomeric Z-band occurs in pediatric cardiomyopathies: a unifying mechanism for force transmission defect. J Cardiovasc Med (Hagerstown) 2009;10:149-156.

6. Mora M, Di Blasi C, Barresi R, Morandi L, Brambati B, Jarre L, Cornelio F. Developmental expression of dystrophin, dystrophin-associated glycoproteins and other membrane cytoskeletal proteins in human skeletal and heart muscle. Brain Res Dev Brain Res 1996;91:70-82.

7. Chevron MP, Girard F, Claustres M, Demaille J. Expression and subcellular localization of dystrophin in skeletal, cardiac and smooth muscles during the human development. Neuromuscul Disord 1994;4:419-432.

8. Sanger JW, Wang J, Fan Y, White J, Sanger JM. Assembly and dynamics of myofibrils. J Biomed Biotechnol 2010;2010:858606.

9. Reiser PJ, Portman MA, Ning XH, Schomisch Moravec C. Human cardiac myosin heavy chain isoforms in fetal and failing adult atria and ventricles. Am J Physiol Heart Circ Physiol 2001;280:H1814-H1820.

10. Racca AW, Klaiman JM, Pioner JM, Cheng Y, Beck AE, Moussavi-Harami F, Bamshad MJ, Regnier M. Contractile properties of developing human fetal cardiac muscle. J Physiol 2016;594:437-452.

11. Schiaffino S, Rossi AC, Smerdu V, Leinwand LA, Reggiani C. Developmental myosins: expression patterns and functional significance. Skelet Muscle 2015;5:22.

12. Pyle WG, Solaro RJ. At the crossroads of myocardial signaling: the role of Z-discs in intracellular signaling and cardiac function. Circ Res 2004;94:296-305.

13. Macadangdang J, Guan X, Smith AS, Lucero R, Czerniecki S, Childers MK, Mack DL, Kim DH. Nanopatterned human iPSC-based model of a dystrophin-null cardiomyopathic phenotype. Cel Mol Bioeng 2015;8:320-332.

14. Pioner JM, Racca AW, Klaiman JM, Yang KC, Guan X, Pabon L, Muskheli V, Zaunbrecher R, Macadangdang J, Jeong MY, Mack DL, Childers MK, Kim DH, Tesi C, Poggesi C, Murry CE, Regnier M. Isolation and mechanical measurements of myofibrils from human induced pluripotent stem cell-derived cardiomyocytes. Stem Cell Rep 2016;6:885-896.
15. Guan X, Mack DL, Moreno CM, Strande JL, Mathieu J, Shi Y, Markert CD, Wang Z, Liu G, Lawlor MW, Moorefield EC, Jones TN, Fugate JA, Furth ME, Murry CE, Ruohola-Baker H, Zhang Y, Santana LF, Childers MK. Dystrophin-deficient cardiomyocytes derived from human urine: new biologic reagents for drug discovery. Stem Cell Res 2014;12:467-480.

16. Cong L, Ran FA, Cox D, Lin S, Barretto R, Habib N, Hsu PD, Wu X, Jiang W, Marraffini LA, Zhang F. Multiplex genome engineering using CRISPR/Cas systems. Science 2013;339:819-823.

17. Colomo F, Nencini S, Piroddi N, Tesi C, Poggesi C. Calcium dependence of the apparent rate of force generation in single striated muscle myofibrils activated by rapid solution changes. Adv Exp Med Biol 1998;453:373-382.

18. Tesi C, Colomo F, Nencini S, Piroddi N, Poggesi C. Modulation by substrate concentration of maximal shortening velocity and isometric force in single myofibrils from frog and rabbit fast skeletal muscle. J Physiol 1999;516:847-853.

19. Moussavi-Harami F, Razumova MV, Racca AW, Cheng Y, Stempien-Otero A, Regnier M. 2-Deoxy adenosine triphosphate improves contraction in human end-stage heart failure. J Mol Cell Cardiol 2015;79:256-263.

20. Palpant NJ, Pabon L, Roberts M, Hadland B, Jones D, Jones C, Moon RT, Ruzzo WL, Bernstein I, Zheng Y, Murry CE. Inhibition of beta-catenin signaling respecifies anterior-like endothelium into beating human cardiomyocytes. Development 2015; 142:3198-3209.

21. Korte FS, Dai J, Buckley K, Feest ER, Adamek N, Geeves MA, Murry CE, Regnier M. Upregulation of cardiomyocyte ribonucleotide reductase increases intracellular 2 deoxy-ATP, contractility, and relaxation. J Mol Cell Cardiol 2011;51:894-901.

22. Kreutziger KL, Piroddi N, McMichael JT, Tesi C, Poggesi C, Regnier M. Calcium binding kinetics of troponin $C$ strongly modulate cooperative activation and tension kinetics in cardiac muscle. J Mol Cell Cardiol 2011;50:165-174.

23. Ferraz SA, Bassani JW, Bassani RA. Rest-dependence of twitch amplitude and sarcoplasmic reticulum calcium content in the developing rat myocardium. J Mol Cell Cardiol 2001;33:711-722.

24. Gazzerro E, Sotgia F, Bruno C, Lisanti MP, Minetti C. Caveolinopathies: from the biology of caveolin-3 to human diseases. Eur J Hum Genet 2010;18:137-145.

25. Mosqueira M, Zeiger U, Forderer M, Brinkmeier H, Fink RH. Cardiac and respiratory dysfunction in Duchenne muscular dystrophy and the role of second messengers. Med Res Rev 2013;33:1174-1213.

26. Sun N, Yazawa M, Liu J, Han L, Sanchez-Freire V, Abilez OJ, Navarrete EG, Hu S, Wang L, Lee A, Pavlovic A, Lin S, Chen R, Hajjar RJ, Snyder MP, Dolmetsch RE, Butte MJ, Ashley EA, Longaker MT, Robbins RC, Wu JC. Patient-specific induced pluripotent stem cells as a model for familial dilated cardiomyopathy. Sci Transl Med 2012;4:130ra47.

27. Masubuchi N, Shidoh Y, Kondo S, Takatoh J, Hanaoka K. Subcellular localization of dystrophin isoforms in cardiomyocytes and phenotypic analysis of dystrophindeficient mice reveal cardiac myopathy is predominantly caused by a deficiency in full-length dystrophin. Exp Anim 2013;62:211-217.

28. Lin B, Li Y, Han L, Kaplan AD, Ao Y, Kalra S, Bett GC, Rasmusson RL, Denning C, Yang L. Modeling and study of the mechanism of dilated cardiomyopathy using induced pluripotent stem cells derived from individuals with Duchenne muscular dystrophy. Dis Model Mech 2015;8:457-466.

29. Stehle R, Solzin J, lorga B, Poggesi C. Insights into the kinetics of $\mathrm{Ca}^{2+}$-regulated contraction and relaxation from myofibril studies. Pflugers Arch 2009;458:337-357.

30. Sasse S, Brand NJ, Kyprianou P, Dhoot GK, Wade R, Arai M, Periasamy M, Yacoub $\mathrm{MH}$, Barton PJ. Troponin I gene expression during human cardiac development and in end-stage heart failure. Circ Res 1993;72:932-938.

31. Lundy SD, Zhu WZ, Regnier M, Laflamme MA. Structural and functional maturation of cardiomyocytes derived from human pluripotent stem cells. Stem Cells Dev 2013; 22:1991-2002.

32. Jelinkova S, Fojtik P, Kohutova A, Vilotic A, Marková L, Pesl M, Jurakova T, Kruta M, Vrbsky J, Gaillyova R, Valášková I, Frák I, Lacampagne A, Forte G, Dvorak P, Meli AC, Rotrekl V. Dystrophin deficiency leads to genomic instability in human pluripotent stem cells via NO synthase-induced oxidative stress. Cells 2019;8.pii:E53.

33. Zhan H, Stanciauskas R, Stigloher C, Dizon KK, Jospin M, Bessereau JL, Pinaud F. In vivo single-molecule imaging identifies altered dynamics of calcium channels in dystrophin-mutant C. elegans. Nat Commun 2014;5:4974.

34. Parton RG, Way M, Zorzi N, Stang E. Caveolin-3 associates with developing Ttubules during muscle differentiation. J Cell Biol 1997;136:137-154.

35. Song KS, Scherer PE, Tang Z, Okamoto T, Li S, Chafel M, Chu C, Kohtz DS, Lisanti MP. Expression of caveolin-3 in skeletal, cardiac, and smooth muscle cells. Caveolin-3 is a component of the sarcolemma and co-fractionates with dystrophin and dystrophin-associated glycoproteins. J Biol Chem 1996;271:15160-15165.

36. Bosman A, Sartiani L, Spinelli V, Del Lungo M, Stillitano F, Nosi D, Mugelli A, Cerbai $\mathrm{E}$, Jaconi M. Molecular and functional evidence of $\mathrm{HCN} 4$ and caveolin-3 interaction during cardiomyocyte differentiation from human embryonic stem cells. Stem Cells Dev 2013;22:1717-1727.

37. Sartiani L, Bettiol E, Stillitano F, Mugelli A, Cerbai E, Jaconi ME. Developmental changes in cardiomyocytes differentiated from human embryonic stem cells: a molecular and electrophysiological approach. Stem Cells 2007;25:1136-1144.

38. Lorin C, Gueffier M, Bois P, Faivre JF, Cognard C, Sebille S. Ultrastructural and functional alterations of EC coupling elements in $\mathrm{mdx}$ cardiomyocytes: an analysis from membrane surface to depth. Cell Biochem Biophys 2013;66:723-736. 
39. Finsterer J, Stollberger C. The heart in human dystrophinopathies. Cardiology 2003; 99:1-19.

40. Merrick D, Stadler LK, Larner D, Smith J. Muscular dystrophy begins early in embryonic development deriving from stem cell loss and disrupted skeletal muscle formation. Dis Model Mech 2009;2:374-388.

41. Wasala NB, Bostick B, Yue Y, Duan D. Exclusive skeletal muscle correction does not modulate dystrophic heart disease in the aged $\mathrm{mdx}$ model of Duchenne cardiomyopathy. Hum Mol Genet 2013;22:2634-2641.

42. Zaunbrecher R, Regnier M. Connecting sarcomere protein mutations to pathogenesis in cardiomyopathies: the development of "disease in a dish" models. Front Physiol 2016;7:566.
43. Passamano L, Taglia A, Palladino A, Viggiano E, D’Ambrosio P, Scutifero M, Rosaria Cecio M, Torre V, F DEL, Picillo E, Paciello O, Piluso G, Nigro G, Politano L. Improvement of survival in Duchenne muscular dystrophy: retrospective analysis of 835 patients. Acta Myol 2012;31:121-125.

44. Markham LW, Michelfelder EC, Border WL, Khoury PR, Spicer RL, Wong BL, Benson DW, Cripe LH. Abnormalities of diastolic function precede dilated cardiomyopathy associated with Duchenne muscular dystrophy. J Am Soc Echocardiogr 2006; 19:865-871.

45. Su JA, Ramos-Platt L, Menteer J. Left ventricular tonic contraction as a novel biomarker of cardiomyopathy in Duchenne muscular dystrophy. Pediatr Cardiol 2016;37: $678-685$. 